\title{
MODULAR GROUPS, HURWITZ CLASSES AND DYNAMIC PORTRAITS OF NET MAPS
}

\author{
WILLIAM FLOYD, WALTER PARRY, AND KEVIN M. PILGRIM
}

\begin{abstract}
An orientation-preserving branched covering $f: S^{2} \rightarrow S^{2}$ is a nearly Euclidean Thurston (NET) map if each critical point is simple and its postcritical set has exactly four points. Inspired by classical, non-dynamical notions such as Hurwitz equivalence of branched covers of surfaces, we develop invariants for such maps. We then apply these notions to the classification and enumeration of NET maps. As an application, we obtain a complete classification of the dynamic critical orbit portraits of NET maps.
\end{abstract}

\section{Contents}

1. Introduction

The translation subgroup

Applications

Outline

Acknowledgements

2. Background

3. Modular group actions 6

4. The extended modular group action on Teichmüller space 10

5. Hurwitz classes and actions of modular groups 13

6. Hurwitz invariants and elementary divisors 16

7. Computing the virtual multi-endomorphism 21

8. Branch data 23

9. Dynamic portraits 24

10. Explicit construction of the dynamic portrait of a NET map 27

11. Explicit construction of NET maps from a dynamic portrait 29

References

\section{INTRODUCTION}

This paper is part of our program to investigate nearly Euclidean Thurston (NET) maps. A Thurston map is NET if each critical point is simple and it has exactly four postcritical points.

The program began with [4], written with Jim Cannon. There, we showed that each NET map $f$ admits a factorization $f=h \circ g$ where $g$ is a Euclidean Thurston map which is affine in suitable coordinates and $h$ is a homeomorphism. This property motivated the

Date: March 14, 2017.

2010 Mathematics Subject Classification. Primary: 36F10; Secondary: 57M12.

Key words and phrases. Thurston map, virtual endomorphism, branched covering. 
term nearly Euclidean. Thus NET maps are among the simplest Thurston maps, and their associated Teichmüller spaces have complex dimension 1. In [4], we exploited the connection to affine geometry to give effective algorithms for the computation of fundamental dynamic invariants, such as the pullback function on homotopy classes of simple closed curves.

Next, 7] shows that every NET map has a presentation which allows it to be described by a simple diagram, such as that shown in Figures 5, 8, and 13, and gives examples showing how to find such diagrams in concrete cases. The data in the diagram can then be translated into input for a computer program, NETmap (see [16]), which implements the algorithms to compute the invariants. The NET map website [15] contains an overview, papers, many examples, and executable files for the computer program NETmap.

The survey [6], written jointly with G. Kelsey, S. Koch, R. Lodge, and E. Saenz, reports on findings and new phenomena from a study of the data generated and on connections to other areas.

This paper develops other invariants of NET maps, focusing on non-dynamical invariants.

We now list these invariants and outline our first main results, in roughly decreasing order of sensitivity. Suppose $f, g$ are Thurston maps with postcritical sets $P_{f}, P_{g}$. In the first four items below, $\phi, \psi$ are orientation-preserving homeomorphisms of the sphere to itself.

- We recall that $f, g$ are Thurston equivalent if there exist such $\phi, \psi$, with $\phi\left(P_{f}\right)=$ $\psi\left(P_{f}\right)=P_{g}, g \circ \psi=\phi \circ f,\left.\phi\right|_{P_{f}}=\left.\psi\right|_{P_{f}}$, and $\phi, \psi$ are isotopic relative to $P_{f}$. Put another way, $f$ and $g$ are conjugate up to isotopy relative to their postcritical sets.

- We say $f, g$ are Hurwitz equivalent for the pure modular group if there exist such $\phi, \psi$ with $\phi\left(P_{f}\right)=\psi\left(P_{f}\right)=P_{g}, g \circ \psi=\phi \circ f$, and $\left.\phi\right|_{P_{f}}=\left.\psi\right|_{P_{f}}$. The motivation for the terminology: pre- and/or post-composing $f$ with a homeomorphism fixing $P_{f}$ pointwise yields a map $g$ which is equivalent to $f$. In suitable Euclidean coordinates, the pure modular group is $\operatorname{PMod}\left(S^{2}, P_{f}\right)=\bar{\Gamma}(2)<P S L(2, \mathbb{Z})$.

- We say $f, g$ are Hurwitz equivalent for the modular group if there exist such $\phi, \psi$ with $\phi\left(P_{f}\right)=\psi\left(P_{f}\right)=P_{g}$ and $g \circ \psi=\phi \circ f$. Theorem 6.1 gives a complete algebraic invariant of the modular group Hurwitz type. In suitable Euclidean coordinates, the modular group is $\operatorname{Mod}\left(S^{2}, P_{f}\right)=P S L(2, \mathbb{Z}) \ltimes(\mathbb{Z} / 2 \mathbb{Z})^{2}$.

- We say $f, g$ are topologically equivalent if there exist such $\phi, \psi$ with $g \circ \psi=\phi \circ f$.

- Every NET map $f$ is topologically equivalent to a Euclidean NET map $g$ induced by a linear map $x \mapsto A x$, where $A$ is a diagonal matrix with entries $(m, n)$ and $n \mid m$. The pair $(m, n)$ are the elementary divisors of $f$. Theorem 6.7 shows that the elementary divisors form a complete invariant of the topological equivalence class of a NET map; see also Theorem 6.8. Hence all NET maps in a given modular group Hurwitz class have the same elementary divisors.

- Most NET maps have four critical values. There are two atypical cases: elementary divisors $(2,1)$ and $(2,2)$. Maps with these elementary divisors have two and three critical values, respectively. In atypical cases, modular group Hurwitz classes may contain both NET and non-NET maps: the postcritical sets of some maps may have fewer than four points. In typical cases, that is, all other cases, modular group Hurwitz classes consist entirely of NET maps. 


\begin{tabular}{|c|c|c|c|c|c|c|c|c|c|c|}
\hline$d$ & 2 & 3 & & & 5 & 6 & 7 & g & & 9 \\
\hline$(m, n)$ & $(2,1)$ & $(3,1)$ & $(4,1)$ & $(2,2)$ & $(5,1)$ & $(6,1)$ & $(7,1)$ & $(8,1)$ & $(4,2)$ & $(9,1)$ \\
\hline$N$ & 3 & 9 & 24 & 8 & 25 & 88 & 47 & 133 & 85 & 120 \\
\hline
\end{tabular}

TABLE 1. The number $N$ of modular group Hurwitz classes of NET maps with elementary divisors $(m, n)$ through degree $d=9$

- Given $y \in S^{2}$, the collection $\{\operatorname{deg}(f, x): f(x)=y\}$ of local degrees defines a partition of $\operatorname{deg}(f)$. The branch data associated to $f$ is the set of such partitions as $y$ varies in the set of branch values of $f$. Section 8 gives a classification of the branch data associated to NET maps. There are three basic types. In even degrees, in addition to the obvious Riemann-Hurwitz condition, there is an exceptional condition: for branch data of type 3, the degree must be divisible by 4 [17, Thm. 3.8].

The translation subgroup. The modular group of the four-times marked sphere $\left(S^{2}, P_{f}\right)$ contains a distinguished subgroup isomorphic to $(\mathbb{Z} / 2 \mathbb{Z})^{2}$. In suitable Euclidean coordinates, its elements are induced by translations. This group acts trivially on the Teichmüller space modelled on $\left(S^{2}, P_{f}\right)$. Suppose $h:\left(S^{2}, P_{f}\right) \rightarrow\left(S^{2}, P_{f}\right)$ represents an element of this group and $g=f \circ h$. Then many fundamental invariants of $f$ and $g$ coincide, though their dynamic portraits may be different. It may also happen that $g=f$, i.e. $h$ is a deck transformation of the covering induced by $f$. This creates some subtleties; $\S 3$ gives a thorough analysis.

Applications. Our first application is to the enumeration of representatives of NET map modular group Hurwitz classes. The website [15] contains data organized first by degree, then by elementary divisors, and finally by modular group Hurwitz class, giving one representative for every modular group Hurwitz class through degree 30. Table 1 gives the numbers of modular group Hurwitz classes of NET maps through degree 9 . For every choice $(m, n)$ of elementary divisors, one modular group Hurwitz class of NET maps consists of all Euclidean NET maps with elementary divisors $(m, n)$.

We now turn to dynamical applications. Suppose $f$ is a Thurston map with set of critical points $C_{f}$ and postcritical set $P_{f}$. The most rudimentary dynamical invariant of $f$ is its dynamic portrait. This invariant records the restriction $f: C_{f} \cup P_{f} \rightarrow P_{f}$ and the local degree $\operatorname{deg}(f, x)$ for $x \in C_{f} \cup P_{f}$. The website [15] contains data organized by degree and dynamic portrait, giving one representative for every dynamic portrait through degree 40. Table 2 gives a precise count of the number of such NET map dynamic portraits in each degree. Theorem 9.2 characterizes when a given pair consisting of elementary divisors $(m, n)$ and a dynamic portrait $\Gamma$ is realizable by a NET map. We also present algorithms for computing the correspondence between dynamic portraits of NET maps and NET map presentations. We describe these algorithms in Sections 10 and 11.

As an example of how non-dynamical invariants influence dynamical properties, we present the following. Below, a Thurston map $f$ is Böttcher expanding if away from periodic critical points, it is uniformly expanding with respect to a natural orbifold metric on the orbifold associated to $f$. Any rational Thurston map is Böttcher expanding.

Theorem 1.1. Suppose $f$ is a non-Euclidean NET map with elementary divisors $(m, n)$ and postcritical set $P_{f}$. If $n>1$ then $f$ is isotopic relative to $P_{f}$ to a Böttcher expanding map. 
The assumption that $f$ is not Euclidean is necessary: if $f$ is the map induced by the matrix $A=2 \cdot\left[\begin{array}{ll}2 & 1 \\ 1 & 1\end{array}\right]$, then $n=2$ while $A$ has a positive real eigenvalue which is strictly less than one; this is an obstruction to expansion in the isotopy class. There are plenty of Böttcher expanding NET maps with $n=1$; among them are many rational maps too, e.g. the Douady rabbit polynomial. So the condition is far from sharp.

Proof. Recall that $f$ is Levy-free if there is no simple closed curve $\gamma \subseteq S^{2}-P_{f}$ which is essential, not peripheral and having a lift $\delta$ homotopic to $\gamma$ which $f$ maps to $\gamma$ with degree 1 . Using Theorem 4.1 of [4], we can see that the degree with which $f$ maps $\delta$ to $\gamma$ is divisible by $n>1$. So $f$ is Levy-free. By [1, Theorem C], $f$ is isotopic to a Böttcher expanding map.

If a modular group Hurwitz class $\mathcal{H}$ contains a non-Euclidean NET map, then every map in $\mathcal{H}$ is non-Euclidean and we call $\mathcal{H}$ non-Euclidean. Any two maps in $\mathcal{H}$ have the same elementary divisors.

Corollary 1.2. Suppose $\mathcal{H}$ is a non-Euclidean modular group Hurwitz class of NET maps and the elementary divisor $n$ is strictly larger than one. Then every isotopy class in $\mathcal{H}$ contains a Böttcher expanding representative.

As another application, we give an effective algorithm for calculating a basic dynamical invariant. Suppose $f:\left(S^{2}, P_{f}\right) \rightarrow\left(S^{2}, P_{f}\right)$ is a NET map with postcritical set $P_{f}$. If a homeomorphism $\psi$ represents an element of $\operatorname{PMod}\left(S^{2}, P_{f}\right)$ and lifts under $f$ to a map $\widetilde{\psi}$ which again represents an element of $\operatorname{PMod}\left(S^{2}, P_{f}\right)$, the assignment $\psi \mapsto \widetilde{\psi}$ induces the virtual endomorphism on the pure mapping class group associated to $f$.

For generic Thurston maps, this is difficult to compute, even for maps with $\# P_{f}=4$. One method is the following. As shown by S. Koch [10], a Thurston map $f$ has an associated algebraic correspondence $X, Y: \mathcal{W} \rightarrow \mathcal{M}$ on the moduli space $\mathcal{M}$ of conformal configurations of embeddings of $P_{f}$ into the Riemann sphere. Here, $Y$ is a finite unramified covering map, and $X$ is holomorphic. If $\# P_{f}=4$, in suitable coordinates, $\mathcal{M}=\mathbb{C}-\{0,1\}, \operatorname{PMod}\left(S^{2}, P_{f}\right)$ is identified with the fundamental group of $\mathcal{M}, \mathcal{W}$ is a compact Riemann surface minus a finite set of points and is given as a locus $\mathcal{W}:=\{P(x, y)=0: x, y \in \mathcal{M}\} \subset \mathbb{C}^{2}$, and the maps $X, Y$ are the coordinate projections. In the special case when $f$ is rational (for ease of exposition here), there is a distinguished "fixed" point $w_{0} \in \mathcal{W}$ with $m_{0}:=X\left(w_{0}\right)=Y\left(w_{0}\right)$, and the virtual endomorphism is then the induced map on fundamental group $X_{*} \circ Y_{*}^{-1}$. In cases with few critical points, such as those considered in [2] and [12], the defining equation $P(x, y)=0$ for the correspondence can be found explicitly, and from this the virtual endomorphism may be computed. For maps with many critical points, this quickly becomes computationally intractable.

We consider a slightly more general notion, and calculate it using different methods. We relax the condition that the homeomorphisms fix $P$ pointwise to merely fixing $P$ setwise, and we obtain an associated virtual multi-endomorphism: the lift $\widetilde{\psi}$ might not be welldefined, due to the possible presence of deck transformations. Theorem 5.2 gives a method for calculating $\psi \rightarrow \widetilde{\psi}$ from knowledge of the slope function $\mu_{f}: \overline{\mathbb{Q}} \rightarrow \overline{\mathbb{Q}}$; see $\$ 2$. This method is then implemented in the NETmap program. Our method relies on two ingredients. First, there is a simple finite linear-algebraic condition for an element $\psi \in \operatorname{Mod}\left(S^{2}, P_{f}\right)$ to be liftable under $f$, i.e. to lie in the domain of the virtual multi-endomorphism (Lemma 3.3). To calculate its image $\widetilde{\psi}$, we exploit two facts: (i) the linear part of an element of $\operatorname{Mod}\left(S^{2}, P_{f}\right)$ is uniquely determined by its value at a pair of distinct extended rationals 
$p / q, r / s \in \mathbb{Q} \cup\{1 / 0\}=: \overline{\mathbb{Q}}$, and (ii) the slope function $\mu_{f}$ is algorithmically computable [4]. Section 7 illustrates this in a complicated example.

As a further application, we obtain information about the geometry of the correspondence $X, Y: \mathcal{W} \rightarrow \mathcal{M}$. The NETmap program uses classical geometric methods to find explicit matrix generators for the domain of the virtual endomorphism (equivalently, for the Fuchsian group that uniformizes $\mathcal{W}$ ), and their images under the virtual endomorphism. The lifting of complex structures under $f$ defines an analytic self-map $\sigma_{f}: \mathbb{H} \rightarrow \mathbb{H}$ on the Teichmüller space modelled on $\left(S^{2}, P_{f}\right)$ that covers the correspondence $X \circ Y^{-1}$. The NETmap program translates algebraic information about the virtual endomorphism into a rather complete geometric description about $\sigma_{f}$; cf. [4]. The data on the website tabulates this geometric information.

Outline. Section 2 collects concepts and facts used throughout the paper. Sections 3 and 4 discuss various modular groups and related actions. Sections 5 and 6 introduce Hurwitz classes, Hurwitz invariants and elementary divisors. Section 7 illustrates the calculation of the virtual endomorphism in a concrete complicated example. Section 8 introduces branch data, used in section 9 to classify dynamic portraits. Sections 10 and 11 give algorithms for constructing a portrait from a map and for constructing maps with a given portrait, respectively.

Acknowledgements. The authors gratefully acknowledge support from the American Institute for Mathematics. Kevin Pilgrim was also supported by Simons grant \#245269.

\section{BACKGROUND}

In this section we summarize some background material from [4] which will be used repeatedly. Let $f: S^{2} \rightarrow S^{2}$ be a NET map. There exist a lattice $\Lambda_{i}$ in $\mathbb{R}^{2}$ and branched covering maps $q_{i}: \mathbb{R}^{2} \rightarrow \mathbb{R}^{2} / 2 \Lambda_{i}, p_{i}: \mathbb{R}^{2} / 2 \Lambda_{i} \rightarrow S^{2}$ and $\pi_{i}=p_{i} \circ q_{i}$ for $i \in\{1,2\}$ so that $f \circ \pi_{1}=\pi_{2}$, as in Figure 1 in Section 1 of [4]. We may always, and usually do, assume $\Lambda_{2}=\mathbb{Z}^{2}$. The postcritical set of $f$ is $P_{2}=\pi_{2}\left(\Lambda_{2}\right)$. Statement 2 of Lemma 1.3 of [4] states that $f^{-1}\left(P_{2}\right)$ contains exactly four points which are not critical points of $f$. This set of four points is $P_{1}=\pi_{1}\left(\Lambda_{1}\right)$. The covering $\pi_{i}$ is normal with group of deck transformations equal to the group $\Gamma_{i}$ of all Euclidean isometries of the form $x \mapsto 2 \lambda \pm x$ for $\lambda \in \Lambda_{i}$ and $i \in\{1,2\}$. We will often use the finite Abelian group $\mathcal{A}:=\Lambda_{2} / 2 \Lambda_{1}$.

We recall Lemmas 2.1 and 2.2 stated in [7].

Lemma 2.1. Let $\Lambda$ and $\Lambda^{\prime}$ be lattices in $\mathbb{R}^{2}$. Let $\Gamma$, respectively $\Gamma^{\prime}$, be the groups of Euclidean isometries of the form $x \mapsto 2 \lambda \pm x$ for some $\lambda \in \Lambda$, respectively $\lambda \in \Lambda^{\prime}$. Also let $\pi: \mathbb{R}^{2} \rightarrow$ $\mathbb{R}^{2} / \Gamma$ and $\pi^{\prime}: \mathbb{R}^{2} \rightarrow \mathbb{R}^{2} / \Gamma^{\prime}$ be the canonical quotient maps. Let $\Phi: \mathbb{R}^{2} \rightarrow \mathbb{R}^{2}$ be an affine isomorphism such that $\Phi(\Lambda) \subseteq \Lambda^{\prime}$. Then $\Phi$ induces a branched covering map $\phi: \mathbb{R}^{2} / \Gamma \rightarrow$ $\mathbb{R}^{2} / \Gamma^{\prime}$ such that $\phi \circ \pi=\pi^{\prime} \circ \Phi$. The map $\Phi$ preserves orientation if and only if $\phi$ preserves orientation. The set $\Phi(\Lambda)$ is a coset of a sublattice $\Lambda^{\prime \prime}$ of $\Lambda^{\prime}$, and the degree of $\phi$ equals the index $\left[\Lambda^{\prime}: \Lambda^{\prime \prime}\right]$.

Lemma 2.2. Let $\Lambda$ and $\Lambda^{\prime}$ be lattices in $\mathbb{R}^{2}$. Let $\Gamma$, respectively $\Gamma^{\prime}$, be the group of Euclidean isometries of the form $x \mapsto 2 \lambda \pm x$ for some $\lambda \in \Lambda$, respectively $\Lambda^{\prime}$. Also let $\pi: \mathbb{R}^{2} \rightarrow \mathbb{R}^{2} / \Gamma$ and $\pi^{\prime}: \mathbb{R}^{2} \rightarrow \mathbb{R}^{2} / \Gamma^{\prime}$ be the canonical quotient maps. Let $\phi: \mathbb{R}^{2} / \Gamma \rightarrow \mathbb{R}^{2} / \Gamma^{\prime}$ be a branched covering map such that $\phi(\pi(\Lambda)) \subseteq \pi^{\prime}\left(\Lambda^{\prime}\right)$. Then we have the following three statements. 
(1) There exists a homeomorphism $\Phi: \mathbb{R}^{2} \rightarrow \mathbb{R}^{2}$ such that the restriction of $\Phi$ to $\Lambda$ is affine and $\phi \circ \pi=\pi^{\prime} \circ \Phi$. If $\pi^{\prime}(0) \in \phi(\pi(\Lambda))$, then $\Phi(\Lambda)$ is a sublattice of $\Lambda^{\prime}$.

(2) There exists an affine isomorphism $\Psi: \mathbb{R}^{2} \rightarrow \mathbb{R}^{2}$ such that the branched map of Lemma 2.1 which $\Psi$ induces from $\mathbb{R}^{2} / \Gamma$ to $\mathbb{R}^{2} / \Gamma^{\prime}$ is $\phi$ up to isotopy rel $\pi(\Lambda)$. If $\pi^{\prime}(0) \in \phi(\pi(\Lambda))$, then $\Psi(\Lambda)$ is a sublattice of $\Lambda^{\prime}$.

(3) The maps $\Phi$ and $\Psi$ are unique up to precomposing with an element of $\Gamma$. They are also unique up to postcomposing with an element of $\Gamma^{\prime}$.

We conclude this section by quickly recalling some dynamical invariants defined by pullback.

Suppose $f$ is a NET map with postcritical set $P_{f}$. The set of (unoriented, simple, essential, non-peripheral, closed, up to free homotopy) curves on $S^{2}-P_{f}$ is naturally identified with $\overline{\mathbb{Q}}:=\mathbb{Q} \cup\left\{\frac{1}{0}\right\}$ via slopes of representing geodesics with respect to the Euclidean metric induced via pushforward under $\pi_{2}$. Pulling back via $f$ induces the slope function $\mu_{f}: \overline{\mathbb{Q}} \rightarrow \overline{\mathbb{Q}} \cup\{\odot\}$, where $\odot$ is a symbol representing the set of inessential and peripheral curves. [4, §5] gives an algorithm for calculating $\mu_{f}$, implemented in the program NETmap.

Let $\gamma$ be a simple closed curve in $S^{2}-P_{f}$ with slope $s \in \overline{\mathbb{Q}}$. The multiplier of both $\gamma$ and $s$ is defined as a fraction $\frac{c}{d}$. The numerator $c$ is the number of connected components of $f^{-1}(\gamma)$ which are neither inessential nor peripheral in $S^{2}-P_{f}$. Theorem 4.1 of [4] shows that the local degrees of $f$ on these connected components are equal. This common local degree is $d$.

The set of marked complex structures on $\left(S^{2}, P_{f}\right)$ is naturally identified with the upper half-plane $\mathbb{H} \subset \mathbb{C}$. We briefly recall this identification. To $\tau \in \mathbb{H}$, one associates the lattice $\Lambda_{\tau}=\mathbb{Z}+\tau \mathbb{Z}$, the group $\Gamma_{\tau}:=\left\{x \mapsto 2 \lambda \pm x, \lambda \in \Lambda_{\tau}\right\}$, and the quotient $X_{\tau}:=\mathbb{C} / \Gamma_{\tau}$ marked at the four points $\Lambda_{\tau} / \Gamma_{\tau}$ and equipped with special generators for its orbifold fundamental group in the obvious way. Conversely, such a quotient equipped with such generators yields a lattice in $\mathbb{C}$ equipped with an ordered basis $\left(\omega_{1}, \omega_{2}\right)$ with $\Im\left(\omega_{2} / \omega_{1}\right)>0$, and this yields an element $\tau:=\omega_{2} / \omega_{1} \in \mathbb{H}$.

Pulling back complex structures on $\left(S^{2}, P_{f}\right)$ under $f$ induces an analytic self-map $\sigma_{f}$ : $\mathbb{H} \rightarrow \mathbb{H}$. The map $\sigma_{f}$ extends continuously to the Weil-Petersson boundary $\overline{\mathbb{Q}}$ so that $\mu_{f}(p / q)=p^{\prime} / q^{\prime}$ if and only if $\sigma_{f}(-q / p)=-q^{\prime} / p^{\prime}$.

\section{Modular GROUP ACTIONS}

This section deals with actions of modular groups on isotopy classes of NET maps. We formally present results only for the modular group. At the end of the section we discuss the analogs for the pure modular group and for the extended modular group, which allows for reversal of orientation. Let $f: S^{2} \rightarrow S^{2}$ be a NET map. This will be fixed for all of this section. As in Section 2, let $\pi_{1}, \pi_{2}: \mathbb{R}^{2} \rightarrow S^{2}$ be branched covering maps so that $f \circ \pi_{1}=\pi_{2}$. The set $P=P_{2}$ of branch values of $\pi_{2}$ in $S^{2}$ is the postcritical set of $f$. Let $\Lambda_{i}$ be the lattice of branch points of $\pi_{i}$ in $\mathbb{R}^{2}$ for $i \in\{1,2\}$.

It is always possible to take the lattice $\Lambda_{2}$ to be $\mathbb{Z}^{2}$. We do so for simplicity. We define the special affine group $\operatorname{SAff}(2, \mathbb{Z})$ to be the group of all orientation-preserving affine isomorphisms $\Psi: \mathbb{R}^{2} \rightarrow \mathbb{R}^{2}$ such that $\Psi\left(\mathbb{Z}^{2}\right)=\mathbb{Z}^{2}$. So $\operatorname{SAff}(2, \mathbb{Z})$ is the set of all maps $x \mapsto A x+b$, where $A \in \mathrm{SL}(2, \mathbb{Z}), x$ is a column vector in $\mathbb{R}^{2}$ and $b$ is a column vector in $\mathbb{Z}^{2}$. As usual, let $\Gamma_{2}$ be the group of all Euclidean isometries of the form $x \mapsto 2 \lambda \pm x$ for some $\lambda \in \mathbb{Z}^{2}$. Let $G$ be the modular group of the pair $\left(S^{2}, P\right)$. Proposition 2.7 of Farb and Margalit's book [5] shows 
that $G$ is a semidirect product with normal subgroup isomorphic to $\Lambda_{2} / 2 \Lambda_{2} \cong(\mathbb{Z} / 2 \mathbb{Z})^{2}$ and quotient group $\operatorname{PSL}(2, \mathbb{Z})$. This is essentially the content of the following proposition.

Proposition 3.1. The branched map $\pi_{2}$ induces a surjective group homomorphism $S A f f(2, \mathbb{Z}) \rightarrow$ $G$ with kernel $\Gamma_{2}$. Hence $G \cong \operatorname{SAff}(2, \mathbb{Z}) / \Gamma_{2}$.

Proof. We define a group homomorphism $\varphi: \operatorname{SAff}(2, \mathbb{Z}) \rightarrow G$ as follows. Let $\Psi \in \operatorname{SAff}(2, \mathbb{Z})$. Lemma 2.1 with $\Lambda=\Lambda^{\prime}=\mathbb{Z}^{2}$ obtains an orientation-preserving homeomorphism $\psi:\left(S^{2}, P\right) \rightarrow$ $\left(S^{2}, P\right)$ such that $\pi_{2} \circ \Psi=\psi \circ \pi_{2}$. We define $\varphi(\Psi)$ to be the isotopy class of $\psi$ in $G$. Using Lemma 2.2, we see that $\varphi$ is surjective, that it is a group homomorphism and that its kernel is $\Gamma_{2}$. This proves Proposition 3.1 .

Because $\operatorname{SAff}(2, \mathbb{Z})$ consists of maps of the form $x \mapsto A x+b$ with $A \in \operatorname{SL}(2, \mathbb{Z})$ and $b \in \mathbb{Z}^{2}$, Proposition 3.1 implies that every element of $G$ has a $\operatorname{PSL}(2, \mathbb{Z})$-term and a translation term. The translation term is an element of $\mathbb{Z}^{2}$ modulo $2 \mathbb{Z}^{2}$. We say that an element of $G$ is elliptic, parabolic or hyperbolic according to whether its $\operatorname{PSL}(2, \mathbb{Z})$-term is elliptic, parabolic or hyperbolic. (We define reflections and glide reflections similarly when dealing with the extended modular group.) We say that an element of $G$ is a translation if its $\operatorname{PSL}(2, \mathbb{Z})$-term is trivial. In this way every element of the extended modular group has a type, that is, it is either elliptic, parabolic, hyperbolic, a reflection, a glide reflection or a translation.

Let $G_{f}$ denote the subgroup of $G$ consisting of those isotopy classes represented by homeomorphisms $\psi:\left(S^{2}, P\right) \rightarrow\left(S^{2}, P\right)$ for which there exists a homeomorphism $\widetilde{\psi}:\left(S^{2}, P\right) \rightarrow$ $\left(S^{2}, P\right)$ such that $\psi \circ f=f \circ \widetilde{\psi}$. Hence the bottom half of the diagram in Figure 1 is commutative. We call $G_{f}$ the subgroup of liftables in $G$. Let $\Delta=\pi_{1}^{-1}(P)$. We define the special affine group $\mathrm{SAff}(f)$ of $f$ to be the group of all orientation-preserving affine isomorphisms $\Psi: \mathbb{R}^{2} \rightarrow \mathbb{R}^{2}$ such that $\Psi\left(\Lambda_{2}\right)=\Lambda_{2}, \Psi\left(\Lambda_{1}\right)=\Lambda_{1}$ and $\Psi(\Delta)=\Delta$. The group $\operatorname{SAff}(f)$ actually depends on $\Lambda_{2}, \Lambda_{1}$ and $\Delta$ in addition to $f$, but it is well defined up to a conjugation isomorphism. Its elements are affine planar homeorphisms that first descend under the projection $\pi_{1}$ and then further descend under $f$, as in Figure 1 .

Proposition 3.2. The group homomorphism of Proposition 3.1 restricts to a surjective group homomorphism $S A f f(f) \rightarrow G_{f}$. In particular, every pair of homeomorphisms $\psi$ and $\widetilde{\psi}$ stabilizing $P$ as in Figure 1 can be modified by isotopies rel $P$ so that they lift to an affine isomorphism $\Psi$ as in Figure 1 . The kernel of this group homomorphism is $S A f f(f) \cap \Gamma_{2}$. So $G_{f} \cong S A f f(f) /\left(S A f f(f) \cap \Gamma_{2}\right)$.

Proof. Let $\Psi \in \operatorname{SAff}(f)$. Lemma 2.1 obtains homeomorphisms $\psi$ and $\widetilde{\psi}$ as in Figure 1 . Hence the group homomorphism of Proposition 3.1 maps $\operatorname{SAff}(f)$ to $G_{f}$. Statement 2 of Lemma 2.2 proves the second statement of Proposition 3.2, giving surjectivity. The kernel of this restriction group homomorphism is clearly $\operatorname{SAff}(f) \cap \Gamma_{2}$. This proves Proposition 3.2.

The preceding discussion gives a simple necessary and sufficient criterion for an element $\psi$ to lie in $G_{f}$. To prepare for the statement, we introduce some terminology that will also feature in our characterization of modular Hurwitz classes. Recall that we have presented $f$ as in the beginning of $\S 2$; we therefore have lattices $\Lambda_{2}:=\mathbb{Z}^{2}$ and $\Lambda_{1}<\Lambda_{2}$. There is a distinguished subset $\mathcal{H S} \subset \mathcal{A}=\Lambda_{2} / 2 \Lambda_{1}$ given by the image of $\Delta:=\pi_{1}^{-1}\left(P_{2}\right)$ under the natural projection $\Lambda_{2} \rightarrow \Lambda_{2} / 2 \Lambda_{1}$. The subset $\mathcal{H S}$ is the Hurwitz structure set associated to this presentation of $f$. The group $\mathcal{A}$ and the subset $\mathcal{H S}$ may be visualized from a presentation 


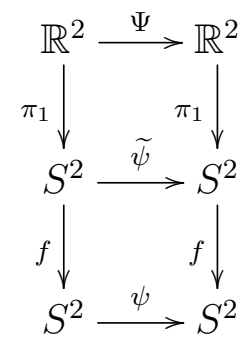

FiguRE 1. Lifting $\psi$; descending $\Psi$

diagram as in Figure 8 as follows. Two copies of the parallelogram form a torus; the grid points in this torus are the elements of $\mathcal{A}$, and the endpoints of the green arcs in this torus are the elements of $\mathcal{H S}$.

Lemma 3.3. Suppose $\Psi \in S A f f(2, \mathbb{Z})$ and let $\psi \in G$ be the induced mapping class element.

(1) $\psi \in G_{f}$ if and only if $\Psi\left(\Lambda_{1}\right)=\Lambda_{1}$ and the induced map $\bar{\Psi}: \mathcal{A} \rightarrow \mathcal{A}$ satisfies $\bar{\Psi}(\mathcal{H S})=\mathcal{H S}$

(2) Suppose $\psi \in G_{f} \cap \operatorname{PMod}\left(S^{2}, P_{f}\right)$. If there exists a lift $\widetilde{\psi}$ which is the identity on $P_{f}$, it is unique. This occurs if and only if $\Psi \in \Gamma(2)+2 \Lambda_{1}, \Psi\left(\Lambda_{1}\right)=\Lambda_{1}$, and the induced map $\bar{\Psi}: \mathcal{A} \rightarrow \mathcal{A}$ has the property that $\bar{\Psi}(h)= \pm h$ for every $h \in \mathcal{H S}$.

Proof. The uniqueness in conclusion 2 follows from [10, Lemma 2.1] as well as the discussion for the pure modular group at the end of this section. The rest is subsumed in the previous discussion.

The next proposition gives some rough bounds on the index of the liftable subgroup.

Proposition 3.4. (1) In terms of the isomorphism $G \cong P S L(2, \mathbb{Z}) \ltimes(\mathbb{Z} / 2 \mathbb{Z})^{2}$, the group $G_{f}$ contains $\bar{\Gamma}(2 D) \times\{0\}$, where $D=\operatorname{deg}(f)$ and $\bar{\Gamma}(2 D)$ is the image in $P S L(2, \mathbb{Z})$ of the principle congruence subgroup $\Gamma(2 D)=\left\{\left[\begin{array}{ll}a & b \\ c & d\end{array}\right] \in S L(2, \mathbb{Z}):\left[\begin{array}{ll}a & b \\ c & d\end{array}\right] \equiv\left[\begin{array}{ll}1 & 0 \\ 0 & 1\end{array}\right] \bmod 2 D\right\}$.

$$
\left[G: G_{f}\right] \leq 16 D^{3} \prod_{p \mid 2 D}\left(1-p^{-2}\right)
$$

Proof. We begin with statement 1 . We have that $D=\left|\Lambda_{2} / \Lambda_{1}\right|$. So $D \Lambda_{2} \subseteq \Lambda_{1}$ and $2 D \Lambda_{2} \subseteq$ $2 \Lambda_{1}$. It follows that $\Gamma(2 D)$ maps $2 \Lambda_{1}$ into itself and acts trivially on $\mathcal{A}$. Hence $\Gamma(2 D) \subseteq$ SAff $(f)$. Proposition 3.2 now completes the proof of statement 1.

Statement 2 results from multiplying $4\left(=\left|(\mathbb{Z} / 2 \mathbb{Z})^{2}\right|\right)$ times the index of $\bar{\Gamma}(2 D)$ in $\operatorname{PSL}(2, \mathbb{Z})$, a well known number [13, Theorem 4.2.5].

The group of modular group deck transformations of $f$ is the set $\operatorname{DeckMod}(f)$ of elements in $G$ represented by homeomorphisms $\varphi:\left(S^{2}, P\right) \rightarrow\left(S^{2}, P\right)$ such that $f \circ \varphi=\psi \circ f$, where $\psi:\left(S^{2}, P\right) \rightarrow\left(S^{2}, P\right)$ is a homeomorphism representing the trivial element of $G$. In particular, if $\psi$ is the identify map, then $\varphi$ is an ordinary deck transformation of $f$ (which stabilizes $P)$. 
Proposition 3.5. (1) The branched map $\pi_{1}$ induces a surjective group homomorphism $S A f f(f) \cap \Gamma_{2} \rightarrow \operatorname{DeckMod}(f)$ with kernel $\Gamma_{1}$. Hence the elements of DeckMod $(f)$ are translations and

$$
\operatorname{DeckMod}(f) \cong\left(S A f f(f) \cap \Gamma_{2}\right) / \Gamma_{1} .
$$

(2) There are three possibilities for DeckMod $(f)$ up to isomorphism:

$$
\{1\}, \mathbb{Z} / 2 \mathbb{Z} \text { or }(\mathbb{Z} / 2 \mathbb{Z})^{2} \text {. }
$$

Proof. Let $\Psi \in \operatorname{SAff}(f) \cap \Gamma_{2}$. Lemma 2.1 shows that $\Psi$ induces homeomorphisms $\psi$ and $\widetilde{\psi}$ as in Figure 1, making the diagram commute. Because $\Psi \in \Gamma_{2}$, the map $\psi$ is the identity map. So $\widetilde{\psi}$ represents an element of $\operatorname{DeckMod}(f)$. This defines a group homomorphism from $\operatorname{SAff}(f) \cap \Gamma_{2}$ to $\operatorname{DeckMod}(f)$. Statement 2 of Lemma 2.2 shows that this homomorphism is surjective. Its kernel is clearly $\operatorname{SAff}(f) \cap \Gamma_{1}=\Gamma_{1}$. This proves statement 1 .

Statement 1 implies that $\operatorname{DeckMod}(f)$ is isomorphic to a subgroup of $\mathcal{A}$. The latter group arises as translations by elements of $2 \Lambda_{2}$ modulo translations by elements of $2 \Lambda_{1}$. Because these translations must map $\Lambda_{1}$ into itself, $\operatorname{DeckMod}(f)$ is in fact isomorphic to a subgroup of $\left(2 \Lambda_{2} \cap \Lambda_{1}\right) / 2 \Lambda_{1} \subseteq \Lambda_{1} / 2 \Lambda_{1} \cong(\mathbb{Z} / 2 \mathbb{Z})^{2}$. This easily proves statement 2 and completes the proof of Proposition 3.5 .

Remark 3.6. In this remark we discuss the fact that each of the three groups in statement 2 of Proposition 3.5 occurs for some NET map. For this we use the notion of Hurwitz structure set defined just before Lemma 3.3. The proof of statement 2 essentially shows that $\operatorname{DeckMod}(f)$ is isomorphic to the subgroup of $\left(2 \Lambda_{2} \cap \Lambda_{1}\right) / 2 \Lambda_{1}$ which stabilizes the Hurwitz structure set $\mathcal{H S}$ of $f$ under the translation action of $\left(2 \Lambda_{2} \cap \Lambda_{1}\right) / 2 \Lambda_{1}$ on $\Lambda_{2} / 2 \Lambda_{1}$. So if $\mathcal{H} \mathcal{S}$ is not a union of cosets of any of the subgroups of order 2 in $\left(2 \Lambda_{2} \cap \Lambda_{1}\right) / 2 \Lambda_{1}$, then $\operatorname{DeckMod}(f)$ is trivial. This is the generic case. In particular, if $2 \Lambda_{2} \cap \Lambda_{1}=2 \Lambda_{1}$, then $\operatorname{DeckMod}(f)$ is trivial. The condition $2 \Lambda_{2} \cap \Lambda_{1}=2 \Lambda_{1}$ is true exactly when the degree $\left|\Lambda_{2} / \Lambda_{1}\right|$ of $f$ is odd. So if the degree of $f$ is odd, then $\operatorname{DeckMod}(f)$ is trivial.

If $\mathcal{H S}$ is a union of cosets of some subgroup of order 2 in $\left(2 \Lambda_{2} \cap \Lambda_{1}\right) / 2 \Lambda_{1}$ but not a larger subgroup of $\left(2 \Lambda_{2} \cap \Lambda_{1}\right) / 2 \Lambda_{1}$, then $\operatorname{DeckMod}(f) \cong \mathbb{Z} / 2 \mathbb{Z}$. This is the situation for the NET map $f$ of Example 10.7 of [4]. In that example we identify $\Lambda_{2} / 2 \Lambda_{1}$ with $(\mathbb{Z} / 4 \mathbb{Z}) \oplus(\mathbb{Z} / 4 \mathbb{Z})$ so that $2 \Lambda_{2}=\Lambda_{1}, \Lambda_{1} / 2 \Lambda_{1}=\{(0,0),(2,0),(0,2),(2,2)\}$ and $\mathcal{H} \mathcal{S}=\{(0,0), \pm(1,0),(2,0), \pm(1,2)\}$. The stabilizer of $\mathcal{H S}$ in $\Lambda_{1} / 2 \Lambda_{1}$ is $\{(0,0),(2,0)\} \cong \mathbb{Z} / 2 \mathbb{Z}$. A nontrivial modular group deck transformation of $f$ is used in [4] to show that $\sigma_{f}$ is constant.

If $\Lambda_{1} \subseteq 2 \Lambda_{2}$ and $\mathcal{H S}$ is a union of cosets of $\Lambda_{1} / 2 \Lambda_{1}$, then $\operatorname{DeckMod}(f) \cong(\mathbb{Z} / 2 \mathbb{Z})^{2}$. This occurs for every Euclidean NET map for which $\Lambda_{1} \subseteq 2 \Lambda_{2}$ because the Euclidean case is exactly the case in which $\mathcal{H} \mathcal{S}=\Lambda_{1} / 2 \Lambda_{1}$.

We next discuss the modular group virtual multi-endomorphism in the present setting. Let $\Psi \in \operatorname{SAff}(f)$. We have two group homomorphisms induced by the assignments $\Psi \mapsto \psi$ and $\Psi \mapsto \widetilde{\psi}$ with $\underset{\sim}{\Psi}, \psi$ and $\widetilde{\psi}$ related as in Figure 1 . Since the diagram in Figure 1 is commutative, $\psi \circ f=f \circ \widetilde{\psi}$. If $\operatorname{DeckMod}(f)$ is trivial, then the assignment $\psi \mapsto \widetilde{\psi}$ determines a group homomorphism from $G_{f}$ to $G$. This is the modular group virtual multi-endomorphism. In general, $\operatorname{DeckMod}(f)$ is nontrivial and instead of the assignment $\psi \mapsto \widetilde{\psi}$ determining a function, it determines a many-valued multifunction. It maps every element of $G_{f}$ to a right coset of DeckMod $(f)$. In particular, the identity element of $G_{f}$ maps to DeckMod $(f)$.

The next proposition shows that these right cosets are also left cosets. It also provides information on the image of translations under the modular group virtual multi-endomorphism. 
Proposition 3.7. (1) The image of $G_{f}$ under the modular group virtual multi-endomorphism is a subgroup of $G$, and DeckMod $(f)$ is a normal subgroup of it.

(2) If the Thurston pullback map $\sigma_{f}$ of $f$ is not constant, then the modular group virtual multi-endomorphism of $f$ maps translations to translations.

Proof. It is easy to see that the image of $G_{f}$ under the modular group virtual multiendomorphism is a subgroup of $G$. To prove the normality statement, let $\delta \in \operatorname{DeckMod}(f)$ and let $\gamma \in G_{f}$. Let $[f]$ denote the isotopy class of $f$, the set of NET maps which are isotopic to $f$ rel the postcritical set of $f$. Then

$$
[f]=[f] \widetilde{\gamma} \widetilde{\gamma}^{-1}=\gamma[f] \widetilde{\gamma}^{-1}=\gamma[f] \delta \widetilde{\gamma}^{-1}=[f] \widetilde{\gamma} \delta \widetilde{\gamma}^{-1} .
$$

Thus $\widetilde{\gamma} \delta \widetilde{\gamma}^{-1} \in \operatorname{DeckMod}(f)$. This proves statement 1 .

To prove statement 2 , let $\varphi$ be a liftable translation in the modular group of $f$. Let $\sigma_{\varphi}$ and $\sigma_{\widetilde{\varphi}}$ be the pullback maps on $\mathbb{H}$ induced by $\varphi$ and $\widetilde{\varphi}$. Then $\sigma_{f} \circ \sigma_{\varphi}=\sigma_{\widetilde{\varphi}} \circ \sigma_{f}$. But $\sigma_{\varphi}=1$ because $\varphi$ is a translation. So $\sigma_{f}=\sigma_{\widetilde{\varphi}} \circ \sigma_{f}$. This implies that $\sigma_{f}$ maps $\mathbb{H}$ into the fixed point set of $\sigma_{\widetilde{\varphi}}$. So if $\sigma_{f}$ is not constant, then $\sigma_{\widetilde{\varphi}}=1$. Hence $\widetilde{\varphi}$ is a translation. This proves Proposition 3.7 .

Remark 3.8. This remark deals with statement 2 of Proposition 3.7. Again let $f$ be the NET map of Example 10.7 of [4]; its pullback map $\sigma_{f}$ is constant. The group $G_{f}$ contains a translation whose image under the modular group virtual multi-endomorphism is elliptic. So translations do not always map to translations.

We continue by considering other choices for the group $G$.

Suppose that $G$ is the pure modular group of the pair $\left(S^{2}, P\right)$. So now the elements of $G$ are isotopy classes of orientation-preserving homeomorphisms $\psi: S^{2} \rightarrow S^{2}$ which fix $P$ pointwise. We consider the analog of Proposition 3.1 in this situation. Since $P$ pulls back to $\Lambda_{2}$ via $\pi_{2}$, the group $G$ pulls back to the elements of $\operatorname{SAff}(2, \mathbb{Z})$ which fix the elements of $\Lambda_{2}$ modulo $\Gamma_{2}$. It follows that $G \cong \bar{\Gamma}(2)=\Gamma(2) /\{ \pm 1\}$, where $\Gamma(2)$ is the subgroup of $\operatorname{SL}(2, \mathbb{Z})$ consisting of those elements which are congruent to 1 modulo 2 . We next consider Proposition 3.2 in this situation. In order for the map $\psi$ in Figure 1 to be in the pure modular group, we need the map $\Psi$ to be in the subgroup generated by $\Gamma(2)$ and $2 \Lambda_{2}$. In order for $\widetilde{\psi}$ to be in the pure modular group, we need $\Psi$ to be in $\operatorname{SAff}_{P}(f)$, which consists of those elements of $\operatorname{SAff}(f)$ which not only map $\Delta$ to itself, but fix the elements of $\Delta$ modulo $2 \Lambda_{1}$. So $G_{f}$ is isomorphic to $\operatorname{SAff}_{P}(f) \cap \bar{\Gamma}(2)$. Using Proposition 3.5, we see that the subgroup of $G$ analogous to $\operatorname{DeckMod}(f)$ is trivial. Because of this, we have a pure modular group virtual endomorphism, a single valued group homomorphism.

In this paragraph we briefly discuss the situation in which we allow for reversal of orientation. So now the elements of $G$ are isotopy classes of homeomorphisms $\psi:\left(S^{2}, P\right) \rightarrow\left(S^{2}, P\right)$ which need not preserve orientation. The analog of Proposition 3.1 is that $G \cong \operatorname{Aff}(2, \mathbb{Z}) / \Gamma_{2}$, where $\operatorname{Aff}(2, \mathbb{Z})$ is defined in the straightforward way. Similarly, Proposition 3.2 becomes $G_{f} \cong \operatorname{Aff}(f) /\left(\operatorname{Aff}(f) \cap \Gamma_{2}\right)$. Finally, we note that the virtual multi-endomorphism is also meaningful in the present situation and that Proposition 3.7 holds as stated in the present situation.

\section{The extended modular group action on Teichmüller SPACE}

In this section we fix a NET map $f$ with postcritical set $P$. Let $G$ denote the extended modular group of the pair $\left(S^{2}, P\right)$. As at the beginning of Section 3 , we represent elements 
$\psi$ of $G$ by affine isomorphisms $\Psi: \mathbb{R}^{2} \rightarrow \mathbb{R}^{2}$ such that $\Psi\left(\mathbb{Z}^{2}\right)=\mathbb{Z}^{2}$. The elements of $G$ act on the Teichmüller space of $\left(S^{2}, P\right)$ by pulling back complex structures on $S^{2} \backslash P$. We let $\sigma_{\psi}$ denote the map on $\mathbb{H}$ induced by the Teichmüller action of $\psi \in G$. The next proposition relates $\Psi$ and $\sigma_{\psi}$. It presents the content of the second paragraph after Theorem 9.1 of [4].

Proposition 4.1. Let $\psi$ be an element of the extended modular group which is represented by the affine map $\Psi(x)=\left[\begin{array}{ll}a & b \\ c & d\end{array}\right] x+t$ with $\left[\begin{array}{ll}a & b \\ c & d\end{array}\right] \in G L(2, \mathbb{Z})$ and $t \in \mathbb{Z}^{2}$. Then the Teichmüller action of $\psi$ on $\mathbb{H}$ is given by $\sigma_{\psi}(z)=\frac{d z+b}{c z+a}$ if $\psi$ preserves orientation and $\sigma_{\psi}(z)=\frac{d \bar{z}+b}{c \bar{z}+a}$ if $\psi$ reverses orientation.

Corollary 4.2. Every element $\psi$ of the extended modular group which is not a translation has the same type as $\sigma_{\psi}$ (elliptic, parabolic,...).

Proof. Since the type of $\psi$ is determined by trace and determinant, this follows from Proposition 4.1.

Let $\widetilde{\psi}$ denote the image of $\psi$ under the extended modular group virtual multi-endomorphism of $f$ for every $\psi \in G_{f}$. In general, the assignment $\psi \mapsto \widetilde{\psi}$ is a multifunction rather than a single-valued function. However, we have the following proposition.

Proposition 4.3. (1) The assignment $\psi \mapsto \sigma_{\widetilde{\psi}}$ for $\psi \in G_{f}$ is a function, even if the extended modular group virtual multi-endomorphism is not.

(2) The assignment $\sigma_{\psi} \mapsto \sigma_{\tilde{\psi}}$ for $\psi \in G_{f}$ is a group homomorphism.

Proof. We begin with statement 1 . The discussion of the modular group virtual multiendomorphism following Proposition 3.5 shows that in general the extended modular group virtual multi-endomorphism is a multifunction which assigns the elements of a coset of $\operatorname{DeckMod}(f)$ to an element of the extended modular group. Statement 1 of Proposition 3.5 shows that the elements of $\operatorname{DeckMod}(f)$ are translations. So they act trivially on $\mathbb{H}$. Thus the assignment $\psi \mapsto \sigma_{\widetilde{\psi}}$ is a function. This proves statement 1 .

Now we consider statement 2. Let $T$ be the normal subgroup of $G$ which consists of all translations. Proposition 4.1 shows that the map $\psi \mapsto \sigma_{\psi}$ induces an injective group antihomomorphism $]^{1}$ from $G / T$ to $\operatorname{PGL}(2, \mathbb{Z})$. As we saw above, the map from $G$ to $G / T$ eliminates the indeterminacy of the modular group virtual multi-endomorphism. As a result, the map $\sigma_{\psi} \mapsto \sigma_{\widetilde{\psi}}$ for $\psi \in G_{f}$ is a group homomorphism.

This proves Proposition 4.3 .

The next theorem provides information about $\sigma_{\widetilde{\psi}}$ in terms of $\sigma_{\psi}$. We prepare for it with a definition. Under the standard action on the upper half-plane, the subgroup of $\operatorname{PSL}(2, \mathbb{Z})$ which fixes an extended rational number $r$ is a parabolic subgroup. This subgroup is infinite cyclic with a positive generator, that is, a generator which fixes $r$ and moves every other element of $\mathbb{R} \cup\{\infty\}$ in the counterclockwise direction relative to $\mathbb{H}$.

Theorem 4.4. Let $\sigma_{f}: \mathbb{H} \rightarrow \mathbb{H}$ be the Thurston pullback map of $f$. Suppose that $\psi \in G_{f}$. Then the following statements hold.

(1) If $\sigma_{\psi}$ is elliptic, then $\sigma_{\widetilde{\psi}}$ is either trivial or elliptic.

(2) Suppose that $\sigma_{\psi}$ is parabolic. Let $s$ be the slope such that $\sigma_{\psi}$ fixes $-1 / s$. Let $m$ be the multiplier of $s$.

(a) If $m=0$, then $\sigma_{\widetilde{\psi}}$ is either trivial or elliptic.

\footnotetext{
${ }^{1} \sigma_{\psi_{2} \circ \psi_{1}}=\sigma_{\psi_{1}} \circ \sigma_{\psi_{2}}$
} 
(b) Suppose that $m \neq 0$, that $\sigma_{\psi}$ is the nth power of the positive generator of the parabolic subgroup of $P S L(2, \mathbb{Z})$ which fixes $-1 / s$ and that the slope function of $f$ maps slope $s$ to slope $t$. Then $\sigma_{\widetilde{\psi}}$ is the $(m n)$-th power of the positive generator of the parabolic subgroup of $P S L(2, \mathbb{Z})$ which fixes $-1 / t$.

(3) Suppose that $\sigma_{\psi}$ is hyperbolic. If the orbifold of $f$ is hyperbolic, then the translation length of $\sigma_{\widetilde{\psi}}$ is less than the translation length of $\sigma_{\psi}$ and the absolute value of the trace of $\sigma_{\widetilde{\psi}}$ is less than the absolute value of the trace of $\sigma_{\psi}$.

(4) If $\sigma_{\psi}$ is a reflection, then $\sigma_{\widetilde{\psi}}$ is a reflection.

(5) If $\sigma_{\psi}$ is a glide reflection, then $\sigma_{\widetilde{\psi}}$ is either a reflection or a glide reflection. If $\sigma_{\widetilde{\psi}}$ is a glide reflection and the orbifold of $f$ is hyperbolic, then the translation length of $\sigma_{\tilde{\psi}}$ is less than the translation length of $\sigma_{\psi}$, and the absolute value of the trace of $\sigma_{\tilde{\psi}}$ is less than the absolute value of the trace of $\sigma_{\psi}$.

Proof. Statement 2 of Proposition 4.3 shows that assignment $\sigma_{\psi} \mapsto \sigma_{\widetilde{\psi}}$ is a group homomorphism. Hence statement 1 is clear.

To prove statement 2, suppose that $\sigma_{\psi}$ is parabolic. Let $s$ and $m$ be as in statement 2 . Since the pure modular group has finite index in the modular group, there exists a positive integer $k$ such that $\psi^{k}$ is in the pure modular group. It therefore is a Dehn twist about a simple closed curve with slope $s$. Let $m=\frac{c}{d}$, as in Section 2, The proof of Theorem 7.1 of [4] shows that the modular group virtual endomorphism maps $\sigma_{\psi^{d k}}$ to the positive generator of the parabolic subgroup of $\operatorname{PSL}(2, \mathbb{Z})$ which fixes $-1 / t$ raised to the power $c k n$. Hence if $c=0$, then $\sigma_{\widetilde{\psi}}$ is either trivial or elliptic. If $c \neq 0$, then we conclude the proof of statement 2 by taking $(d k)$-th roots.

To prove statement 3, suppose that both $\sigma_{\psi}$ and the orbifold of $f$ are hyperbolic. We prove statement 3 by adapting the proof of Proposition 6.5 of [11] to the present situation. Because the orbifold of $f$ is hyperbolic, Theorem 6.3 of [11] implies that the modular group virtual multi-endomorphism of $f$ is not injective. In fact, its kernel contains a hyperbolic element. Hence $\sigma_{f}$ is not injective. Not being an isometry, it strictly decreases hyperbolic distances. Let $d: \mathbb{H} \times \mathbb{H} \rightarrow \mathbb{R}$ denote a hyperbolic metric on $\mathbb{H}$. Among all elements $z \in \mathbb{H}$, the minimum value of $d\left(z, \sigma_{\psi}(z)\right)$ is achieved exactly for the elements on the translation axis of $\sigma_{\psi}$. It is the translation length of $\sigma_{\psi}$. Let $z$ be an element of the translation axis of $\sigma_{\psi}$, and let $w=\sigma_{f}(z)$. Then

$$
d\left(w, \sigma_{\widetilde{\psi}}(w)\right)=d\left(\sigma_{f}(z), \sigma_{\widetilde{\psi}}\left(\sigma_{f}(z)\right)\right)=d\left(\sigma_{f}(z), \sigma_{f}\left(\sigma_{\psi}(z)\right)\right)<d\left(z, \sigma_{\psi}(z)\right) .
$$

Thus the translation length of $\sigma_{\widetilde{\psi}}$ is less than the translation length of $\sigma_{\psi}$.

We next prove that the absolute value of the trace of $\sigma_{\widetilde{\psi}}$ is less than the absolute value of the trace of $\sigma_{\psi}$. This is clear if $\sigma_{\widetilde{\psi}}$ is not hyperbolic, so we assume that $\sigma_{\widetilde{\psi}}$ is hyperbolic. We use the fact that, up to a global multiplicative constant, the translation length of a hyperbolic element $\gamma$ of $\operatorname{SL}(2, \mathbb{Z})$ is $\ln (|\lambda|)$, where $\lambda$ is the eigenvalue of $\gamma$ with larger absolute value. The absolute value of the trace of $\gamma$ is $\left|\lambda+\lambda^{-1}\right| \in \mathbb{Z}$. So decreasing $|\lambda|$ decreases the absolute value of the trace of $\gamma$. It follows that the absolute value of the trace of $\sigma_{\widetilde{\psi}}$ is less than the absolute value of the trace of $\sigma_{\psi}$. This proves statement 3 .

To prove statement 4 , note that if $\sigma_{\psi}$ is a reflection, then the equation $\sigma_{f} \circ \sigma_{\psi}=\sigma_{\widetilde{\psi}^{\circ}}$. $\sigma_{f}$ implies that $\sigma_{\widetilde{\psi}}$ reverses orientation. Statement 2 of Proposition 4.3 implies that the assignment $\sigma_{\psi} \mapsto \sigma_{\widetilde{\psi}}$ is a group homomorphism. So $\sigma_{\widetilde{\psi}}$ is an involution which reverses orientation. It must be a reflection. 
Statement 5 can be proved much as statements 3 and 4 were proved.

This completes the proof of Theorem 4.4.

As a nice application of the material in this section and the previous one, we prove the following theorem. This theorem easily generalizes to all Thurston maps with four postcritical points. It complements [11, Theorem 9.2], which treats only twisting by full Dehn twists.

Theorem 4.5. Let $f$ be a non-Euclidean NET map with an obstruction whose multiplier is 1. Let $\omega$ be an element in the modular group of $f$ which together with the translations generates the stabilizer of the obstruction of $f$. Then the homotopy classes $[f] \omega^{n}$ are mutually Thurston inequivalent for $n \in \mathbb{Z}$.

Proof. Let $m$ and $n$ be integers such that $[f] \omega^{m}$ and $[f] \omega^{n}$ are Thurston equivalent. Then there exists an element $\varphi$ in the modular group of $f$ such that $\varphi[f] \omega^{m} \varphi^{-1}=[f] \omega^{n}$. Now we use the fact that if $f_{1}$ and $f_{2}$ are obstructed NET maps with $f_{2}=\psi f_{1} \psi^{-1}$, where $\psi$ is a homeomorphism representing an element of the modular group of $f_{1}$, then $\psi$ maps the obstruction of $f_{1}$ to the obstruction of $f_{2}$. Hence $\varphi$ fixes the obstruction of $f$. This and the assumptions imply that $\varphi$ is a power of $\omega$ up to translations, and so $\varphi$ and $\omega$ commute up to translations. Next we note that the equation $\varphi[f] \omega^{m} \varphi^{-1}=[f] \omega^{n}$ implies that $\varphi$ is liftable relative to $f$. So $[f] \widetilde{\varphi} \omega^{m} \varphi^{-1} \omega^{-n}=[f]$, where $\widetilde{\varphi}$ is one modular group element in the image of $\varphi$ under the modular group virtual multi-endomorphism of $f$. Because the

obstruction of $f$ has multiplier 1 , statement 2 of Theorem 4.4 implies that $\widetilde{\varphi}=\varphi \tau$ for some translation $\tau$. Hence $[f] \omega^{m-n} \tau^{\prime}=[f]$ for some translation $\tau^{\prime}$. Hence $\omega^{m-n} \tau^{\prime} \in \operatorname{DeckMod}(f)$. But Proposition 3.5 implies that $\operatorname{DeckMod}(f)$ consists of translations. Thus $m=n$, which proves Theorem 4.5 .

\section{HuRWitz CLASSES AND ACTIONS OF MODUlAR GROUPS}

This section introduces Hurwitz classes of NET maps and considers related actions of modular groups. There are various definitions of Hurwitz equivalence in the literature. We give definitions which are suitable for our study of NET maps.

We first recall some definitions from the introduction, and give some related terminology. Let $f: S^{2} \rightarrow S^{2}$ and $f^{\prime}: S^{2} \rightarrow S^{2}$ be NET maps with postcritical sets $P$ and $P^{\prime}$. Let $\varphi, \psi:\left(S^{2}, P\right) \rightarrow\left(S^{2}, P^{\prime}\right)$ be orientation-preserving homeomorphisms such that $\varphi \circ f=f^{\prime} \circ \psi$. In this situation we say that $f$ and $f^{\prime}$ are Hurwitz equivalent for the modular group. This defines an equivalence relation on the set of NET maps. The equivalence classes of this equivalence relation are called modular group Hurwitz classes. We denote the modular group Hurwitz class of $f$ by $\operatorname{Hurw}(f)$. If in addition $\varphi$ and $\psi$ agree on $P$, then we say that $f$ and $f^{\prime}$ are Hurwitz equivalent for the pure modular group. The equivalence classes of this equivalence relation are called pure modular group Hurwitz classes. We denote the pure modular group Hurwitz class of $f$ by $\operatorname{PHurw}(f)$.

We let $\operatorname{Hurw}_{P}(f)$ denote the subset of $\operatorname{Hurw}(f)$ consisting of those maps $f^{\prime}$ with $P^{\prime}=P$. The group $\operatorname{Homeo}\left(S^{2}, P\right)$ of all orientation-preserving homeomorphisms from $S^{2}$ to $S^{2}$ which map $P$ to itself acts on $\operatorname{Hurw}_{P}(f)$ both on the left and the right. So does the subgroup Homeo $_{0}\left(S^{2}, P\right)$ consisting of those elements which are isotopic rel $P$ to the identity map. We let

$$
\operatorname{HurwMod}_{P}(f)=\operatorname{Homeo}_{0}\left(S^{2}, P\right) \backslash \operatorname{Hurw}_{P}(f) / \operatorname{Homeo}_{0}\left(S^{2}, P\right) \text {. }
$$


We let $[f]$, the isotopy class of $f$, denote the element of $\operatorname{HurwMod}_{P}(f)$ represented by $f$. Similarly,

$$
\operatorname{PHurwMod}_{P}(f)=\operatorname{Homeo}_{0}\left(S^{2}, P\right) \backslash \operatorname{PHurw}_{P}(f) / \operatorname{Homeo}_{0}\left(S^{2}, P\right) .
$$

The modular group $\operatorname{Mod}\left(S^{2}, P\right)$ acts on $\operatorname{HurwMod}_{P}(f)$ both on the left and the right by post- and pre-composition, respectively. Equipped with these commuting actions, $\operatorname{HurwMod}_{P}(f)$ is a $\operatorname{Mod}\left(S^{2}, P\right)$-biset, in the sense of $[14]^{2}$. Since the right action need not be free, the algebraic structure is more complicated. Thus we have a fundamental tension: pure modular group Hurwitz classes behave better algebraically, while modular group Hurwitz classes are in some sense more fundamental.

Just as for NET maps, we let $[\varphi]$ denote the isotopy class rel $P$ of a homeomorphism $\varphi:\left(S^{2}, P\right) \rightarrow\left(S^{2}, P\right)$. We define the left and right stabilizers of $[f]$ in $\operatorname{Mod}\left(S^{2}, P\right)$ in the straightforward way. Similarly, the pure modular group $\operatorname{PMod}\left(S^{2}, P\right)$ acts on $\operatorname{PHurwMod}(f)$ both on the left and the right. We define left and right stabilizers of $[f]$ in $\operatorname{PMod}\left(S^{2}, P\right)$ as before.

There is a modular group virtual multi-endomorphism of $\operatorname{Mod}\left(S^{2}, P\right)$ and a pure modular group virtual endomorphism of $\operatorname{PMod}\left(S^{2}, P\right)$. By the kernel of the modular group virtual multi-endomorphism we mean the set of all isotopy classes in $\operatorname{Mod}\left(S^{2}, P\right)$ for which some image under the modular group virtual multi-endomorphism is trivial.

The following theorem presents some results concerning the action of the modular group on $\operatorname{HurwMod}_{P}(f)$. Essentially the same results hold for pure modular Hurwitz classes. We discuss this very briefly after the proof.

Theorem 5.1. Let $f$ be a NET map with postcritical set $P$. Let $G=\operatorname{Mod}\left(S^{2}, P\right)$, and let $G_{f}$ be the group of liftables in $G$ as usual.

(1) The left stabilizer of $[f]$ in $G$ is the kernel of the modular group virtual multiendomorphism.

(2) The right stabilizer of $[f]$ in $G$ is DeckMod $(f)$.

(3) If $\gamma \in G_{f}$, then $\gamma[f]=[f] \widetilde{\gamma}$, where $\widetilde{\gamma}$ is any image of $\gamma$ under the modular group virtual multi-endomorphism.

(4) Let $\psi_{1}, \ldots, \psi_{k}$ form a left transversal for $G_{f}$ in $G$, and let $\gamma_{1}, \gamma_{2} \in G$. Then $\psi_{i}[f] \gamma_{1}=$ $\psi_{j}[f] \gamma_{2}$ for some $i, j \in\{1, \ldots, k\}$ if and only if $i=j$ and $\gamma_{2} \gamma_{1}^{-1} \in \operatorname{DeckMod}(f)$.

(5) The right action of $G$ on $\operatorname{HurwMod}_{P}(f)$ has finitely many orbits.

(6) Let $\varphi, \psi:\left(S^{2}, P\right) \rightarrow\left(S^{2}, P\right)$ be orientation-preserving homeomorphisms. Then $f \circ \varphi$ is Thurston equivalent to $f \circ \psi$ if and only if $[\varphi]=\widetilde{\gamma}[\psi] \gamma^{-1}$ for some $\gamma \in G_{f}$ and some image $\tilde{\gamma}$ of $\gamma$ under the modular group virtual multi-endomorphism.

Proof. Statements 1, 2 and 3 are clear.

To prove statement 4 , suppose that $\psi_{i}[f] \gamma_{1}=\psi_{j}[f] \gamma_{2}$ for some $i, j \in\{1, \ldots, k\}$. Then $\psi_{j}^{-1} \psi_{i}[f]=[f] \gamma_{2} \gamma_{1}^{-1}$. This means that $\psi_{j}^{-1} \psi_{i} \in G_{f}$. So $i=j$. Hence $[f]=[f] \gamma_{2} \gamma_{1}^{-1}$. This means that $\gamma_{2} \gamma_{1}^{-1} \in \operatorname{DeckMod}(f)$. This easily proves statement 4 .

To prove statement 5, we use the fact that every element of $\operatorname{HurwMod}_{P}(f)$ has the form $\varphi[f] \psi$ for some $\varphi, \psi \in G$. Hence statement 5 follows from statement 3 and statement 2 of Proposition 3.4, which implies that $G_{f}$ has finite index in $G$.

To prove statement 6 , let $\varphi, \psi:\left(S^{2}, P\right) \rightarrow\left(S^{2}, P\right)$ be orientation-preserving homeomorphisms such that $f \circ \varphi$ is Thurston equivalent to $f \circ \psi$. It follows that $[f \circ \varphi]=[f][\varphi]=$

\footnotetext{
${ }^{2}$ The term 'permutational bimodule' is used there instead of 'biset'.
} 
$\gamma[f \circ \psi] \gamma^{-1}=\gamma[f][\psi] \gamma^{-1}$ for some $\gamma \in G$. This easily implies that $\gamma \in G_{f}$. Now statement 3 implies that $[f][\varphi]=[f] \widetilde{\gamma}[\psi] \gamma^{-1}$. Now Statement 4 implies that there exists $\epsilon \in \operatorname{DeckMod}(f)$ such that $\widetilde{\gamma}[\psi] \gamma^{-1}[\varphi]^{-1}=\epsilon$. So $[\varphi]=\epsilon^{-1} \widetilde{\gamma}[\psi] \gamma^{-1}$. Since $\epsilon^{-1} \widetilde{\gamma}$ is an image of $\gamma$ under the modular group virtual multi-endomorphism, this proves the forward implication of statement 6. The backward implication is now clear.

This proves Theorem 5.1 .

Theorem 5.1 is essentially valid when $\operatorname{Mod}\left(S^{2}, P\right)$ is replaced by $\operatorname{PMod}\left(S^{2}, P\right)$ and $\operatorname{HurwMod} \operatorname{Mod}_{P}(f)$ is replaced by PHurwMod $P(f)$. In statements 2 and 4 the group $\operatorname{DeckMod}(f)$ is replaced by the trivial group. Statement 6 applied to $\operatorname{Mod}\left(S^{2}, P\right)$ already handles the case in which $\varphi$ and $\psi$ represent elements of $\operatorname{PMod}\left(S^{2}, P\right)$ : even if $\varphi$ and $\psi$ represent elements of $\operatorname{PMod}\left(S^{2}, P\right)$, all that can be said about the element $\gamma$ is that it represents an element of $\operatorname{Mod}\left(S^{2}, P\right)$.

The next theorem provides an effective procedure for calculating the modular group virtual multi-endomorphism. Effectiveness comes from the fact that the slope function $\mu_{f}$ is effectively computable [4, §5].

Theorem 5.2. Let $f$ be a NET map, and suppose that the usual lattice $\Lambda_{2}$ is $\mathbb{Z}^{2}$. We calculate slopes of simple closed curves in $S^{2} \backslash P_{2}$ using the standard basis vectors $e_{1}$ and $e_{2}$ of $\mathbb{Z}^{2}$ as usual. Let $\mu_{f}$ be the slope function of $f$ and suppose that there exist slopes $s$ and $t$ such that $\mu_{f}(s)$ and $\mu_{f}(t)$ are distinct slopes. Let $\Psi \in A f f(f)$, and let $\widetilde{\psi}: S^{2} \rightarrow S^{2}$ and $\psi: S^{2} \rightarrow S^{2}$ be the homeomorphisms induced by $\pi_{1}$ and $\pi_{2}$ as in Figure 1 . Let $\widetilde{\Psi}: \mathbb{R}^{2} \rightarrow \mathbb{R}^{2}$ be an affine isomorphism such that the homeomorphism of $S^{2}$ which $\widetilde{\Psi}$ induces on $S^{2}$ via $\pi_{2}$ is isotopic to $\widetilde{\psi}$ rel $P_{2}$.

(1) The linear part $x \mapsto Q x$ of $\widetilde{\Psi}$ can be computed as follows. Suppose that $\Psi$ takes lines with slope $s$, respectively $t$, to lines with slope $s^{\prime}$, respectively $t^{\prime}$. Then $Q$ is the element of $G L(2, \mathbb{Z})$, unique up to multiplication by \pm 1 whose determinant equals the determinant of $\Psi$ and which takes lines with slope $\mu_{f}(s)$, respectively $\mu_{f}(t)$, to lines with slope $\mu_{f}\left(s^{\prime}\right)$, respectively $\mu_{f}\left(t^{\prime}\right)$.

(2) The translation part of $\widetilde{\Psi}$ can be computed as follows. Let $T=\left\{0, e_{1}, e_{2}, e_{1}+e_{2}\right\}$, and for $\tau \in T$ let $x_{\tau}=\pi_{2}(\tau)$. So the postcritical set of $f$ is $\left\{x_{0}, x_{e_{1}}, x_{e_{2}}, x_{e_{1}+e_{2}}\right\}$. Then the translation term of $\widetilde{\Psi}$ is the element $\tau \in T$ for which $\Psi$ maps $\pi_{1}^{-1}\left(x_{0}\right)$ to $\pi_{1}^{-1}\left(x_{\tau}\right)$.

Proof. We first prove statement 1 . Let $\varphi_{s}:\left(S^{2}, P_{2}\right) \rightarrow\left(S^{2}, P_{2}\right)$ be a Dehn twist (possibly twisting many times) about a simple closed curve in $S^{2} \backslash P_{2}$ with slope $s$. Since $\Psi$ takes lines with slope $s$ to lines with slope $s^{\prime}$, the homeomorphism $\varphi_{s^{\prime}}=\psi \circ \varphi_{s} \circ \psi^{-1}$ is a Dehn twist about a simple closed curve in $S^{2} \backslash P_{2}$ with slope $s^{\prime}$. We next apply the argument which immediately precedes Theorem 7.1 of [4]. This argument shows that the modular group virtual multi-endomorphism maps the isotopy class of some power of $\varphi_{s}$ to the isotopy class of a Dehn twist $\widetilde{\varphi}_{s}$ (possibly twisting many times) about a simple closed curve in $S^{2} \backslash P_{2}$ with slope $\mu_{f}(s)$. A corresponding statement holds for $\varphi_{s^{\prime}}$. We replace $\varphi_{s}$ and $\varphi_{s^{\prime}}$ by appropriate powers to obtain the diagram in Figure 2. Thus $\widetilde{\psi}$ takes simple closed curves in $S^{2} \backslash P_{2}$ with slope $\mu_{f}(s)$ to simple closed curves in $S^{2} \backslash P_{2}$ with slope $\mu_{f}\left(s^{\prime}\right)$. So $\widetilde{\Psi}$, equivalently $Q$, takes lines with slope $\mu_{f}(s)$ to lines with slope $\mu_{f}\left(s^{\prime}\right)$. Similarly, $Q$ takes lines with slope $\mu_{f}(t)$ to lines with slope $\mu_{f}\left(t^{\prime}\right)$. Because $f$ preserves orientation, it is clear that $Q$ must have determinant 1 if $\Psi$ preserves orientation and it must have determinant -1 if $\Psi$ reverses orientation. 


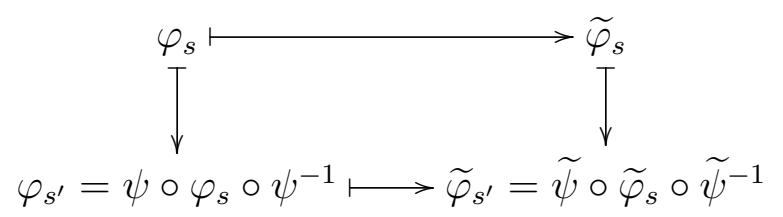

Figure 2. Proving Theorem 5.2

To see that this determines $Q$ up to multiplication by \pm 1 , let $R$ be an element of $\mathrm{GL}(2, \mathbb{Z})$ with $\operatorname{det}(R)=\operatorname{det}(Q)$ which takes lines with slope $\mu_{f}(s)$, respectively $\mu_{f}(t)$, to lines with slope $\mu_{f}\left(s^{\prime}\right)$, respectively $\mu_{f}\left(t^{\prime}\right)$. Then $R^{-1} Q$ takes lines with slope $\mu_{f}(s)$, respectively $\mu_{f}(t)$, to lines with slope $\mu_{f}(s)$, respectively $\mu_{f}(t)$. This means that the linear fractional transformation associated to $R^{-1} Q$ arises from an element of $\operatorname{PSL}(2, \mathbb{Z})$ and it fixes two extended rational numbers. Hence this linear fractional transformation is the identity map. This proves statement 1 of Theorem 5.2 .

Statement 2 is rather clear, and so the proof of Theorem 5.2 is complete.

\section{HuRWitz inVARIANTS AND ELEMENTARY DIVISORS}

The main goal of this section is to find a rather simple complete combinatorial invariant for NET map modular group Hurwitz classes. This is achieved in Theorem 6.1. Then we show that elementary divisors form a complete set of invariants for topological equivalence; this is Theorem 6.7.

We begin with a discussion of elementary divisors. Let $f$ be a NET map. From a presentation for $f$ we have lattices $\Lambda_{1} \subseteq \Lambda_{2}$ as usual; we have the group $\mathcal{A}=\Lambda_{2} / 2 \Lambda_{1}$ and the Hurwitz structure set $\mathcal{H S}$ from $\S 3$. There exist unique positive integers $m$ and $n$ with $n \mid m$ such that $\Lambda_{2} / \Lambda_{1} \cong(\mathbb{Z} / m \mathbb{Z}) \oplus(\mathbb{Z} / n \mathbb{Z})$. The integers $m$ and $n$ are the elementary divisors of $f$, defined in Section 8 of [7]. Here is another way to view the elementary divisors of $f$. We factor $f$ in the usual way as $f=h \circ g$, where $g$ is a Euclidean NET map and $h$ is a homeomorphism. The map $g$ is induced by an orientation-preserving affine isomorphism $\Phi: \mathbb{R}^{2} \rightarrow \mathbb{R}^{2}$ such that $\Phi\left(\Lambda_{2}\right)=\Lambda_{1}$. Expressing $\Phi$ in terms of a basis for $\Lambda_{2}$, we have that $\Phi(x)=A x+b$, where $A$ is a $2 \times 2$ matrix of integers and $b \in \Lambda_{1}$. There exist matrices $Q, R \in \operatorname{SL}(2, \mathbb{Z})$ such that $A=Q D R$, where $D$ is the diagonal matrix whose diagonal entries are $m$ and $n$. So $m$ and $n$ are the elementary divisors of $A$. Since $\operatorname{deg}(f)=\operatorname{deg}(g)=\operatorname{det}(A)=m n$, the elementary divisors of $f$ give finer information than $\operatorname{deg}(f)$. We will see in Corollary 6.2 that $m$ and $n$ are uniquely determined by $f$. The atypical cases are $(m, n) \in\{(2,1),(2,2)\}$.

We continue with a discussion of Hurwitz structure sets. The Hurwitz structure set of $f$, defined in Section 3, depends on the presentation. Here, we analyze the dependency. We say that a subset $\mathcal{H} \mathcal{S}$ of $\mathcal{A}$ is a Hurwitz structure set if it is a disjoint union of four subsets of the form $\mathcal{H S}=\left\{ \pm h_{1}\right\} \amalg\left\{ \pm h_{2}\right\} \amalg\left\{ \pm h_{3}\right\} \amalg\left\{ \pm h_{4}\right\}$, where $h_{i} \in \mathcal{A}$. It is possible that $h_{i}=-h_{i}$, but $h_{i} \neq \pm h_{j}$ if $i \neq j$ for $i, j \in\{1,2,3,4\}$. The Hurwitz structure set coming from a NET map presentation gives such an example. In fact, every such set $\mathcal{H} \mathcal{S} \subseteq \mathcal{A}$ is the Hurwitz structure set associated to some NET map. Now suppose that we also have possibly different lattices $\Lambda_{1}^{\prime} \subseteq \Lambda_{2}^{\prime}$. Let $\Psi: \Lambda_{2} \rightarrow \Lambda_{2}^{\prime}$ be an orientation-preserving affine isomorphism such that $\Psi\left(\Lambda_{1}\right)=\Lambda_{1}^{\prime}$. Then $\Psi$ induces an affine bijection $\bar{\Psi}: \mathcal{A} \rightarrow \mathcal{A}^{\prime}$. The linear part of $\bar{\Psi}$ takes Hurwitz structure sets in $\mathcal{A}$ to Hurwitz structure sets in $\mathcal{A}^{\prime}$. Since $\Psi(0) \in \Lambda_{1}^{\prime}$, the translation part of $\bar{\Psi}$ is an element of order 2 . It easily follows that the 


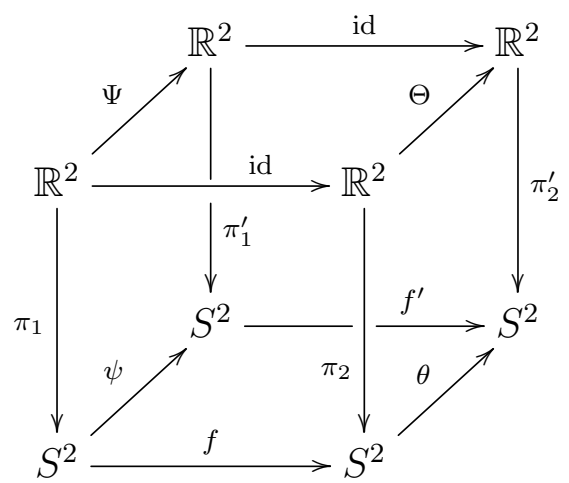

Figure 3. Proving the forward direction of Theorem 6.1

translation part of $\bar{\Psi}$ takes Hurwitz structure sets in $\mathcal{A}^{\prime}$ to Hurwitz structure sets in $\mathcal{A}^{\prime}$. So $\bar{\Psi}$ takes Hurwitz structure sets to Hurwitz structure sets. We say that Hurwitz structure sets $\mathcal{H S} \subseteq \mathcal{A}$ and $\mathcal{H} \mathcal{S}^{\prime} \subseteq \mathcal{A}^{\prime}$ are equivalent if there exists such an affine isomorphism $\Psi: \Lambda_{2} \rightarrow \Lambda_{2}^{\prime}$ with $\Psi\left(\Lambda_{1}\right)=\Lambda_{1}^{\prime}$ such that $\mathcal{H} \mathcal{S}^{\prime}=\bar{\Psi}(\mathcal{H S})$. This defines an equivalence relation on Hurwitz structure sets.

The Hurwitz invariant of $f$ is defined as the equivalence class of the Hurwitz structure set $\mathcal{H S}$ under the equivalence relation of the preceding paragraph. It is a finer invariant than the elementary divisors.

Theorem 6.1. The Hurwitz invariant of a NET map $f$ is an invariant of the modular group Hurwitz class of $f$. Conversely, two modular group Hurwitz classes of NET maps with equal Hurwitz invariants are equal. Thus the Hurwitz invariant is a complete invariant of modular group Hurwitz classes of NET maps.

Proof. We first prove that Hurwitz invariants are indeed invariants of modular group Hurwitz classes. Let $f$ and $f^{\prime}$ be NET maps which are Hurwitz equivalent for the modular group. Let $p_{1}, q_{1}, p_{2}, q_{2}, \ldots$ be the usual maps, lattices, groups and four-element sets for $f$. We likewise have maps, lattices, groups and four-element sets for $f^{\prime}$. Because $f$ and $f^{\prime}$ are Hurwitz equivalent for the modular group, there exist orientation-preserving homeomorphisms $\psi, \theta:\left(S^{2}, P_{2}\right) \rightarrow\left(S^{2}, P_{2}^{\prime}\right)$ such that $\theta \circ f=f^{\prime} \circ \psi$. This implies that $\psi\left(P_{1}\right)=P_{1}^{\prime}$. Now statement 1 of Lemma 2.2 implies that there exist orientation-preserving homeomorphisms $\Psi, \Theta: \mathbb{R}^{2} \rightarrow \mathbb{R}^{2}$ such that $\Psi\left(\Lambda_{1}\right)=\Lambda_{1}^{\prime}, \Theta\left(\Lambda_{2}\right)=\Lambda_{2}^{\prime}$, the restriction of $\Psi$ to $\Lambda_{1}$ is affine, the restriction of $\Theta$ to $\Lambda_{2}$ is affine, $\pi_{1}^{\prime} \circ \Psi=\psi \circ \pi_{1}$ and $\pi_{2}^{\prime} \circ \Theta=\theta \circ \pi_{2}$. Hence every face of the cube in Figure 3 is commutative except possibly the top.

Now we consider the top of the cube in Figure 3. Since every face of the cube other than the top is commutative, $\pi_{2}^{\prime} \circ \Psi=\pi_{2}^{\prime} \circ \Theta$. It follows that $\Psi$ is $\Theta$ postcomposed with a deck transformation for $\pi_{2}^{\prime}$, namely, an element of $\Gamma_{2}^{\prime}$. So $\Psi\left(\Lambda_{2}\right)=\Lambda_{2}^{\prime}$, and the restriction of $\Psi$ to $\Lambda_{2}$ is affine. So $\Psi$ induces an affine bijection $\bar{\Psi}: \mathcal{A} \rightarrow \mathcal{A}^{\prime}$. Since $\psi\left(P_{2}\right)=P_{2}^{\prime}$, the map $\bar{\Psi}$ takes the Hurwitz structure set $p_{1}^{-1}\left(P_{2}\right)$ of $f$ to the Hurwitz structure set $\left(p_{1}^{\prime}\right)^{-1}\left(P_{2}^{\prime}\right)$ of $f^{\prime}$. Thus the Hurwitz invariant of $f$ equals the Hurwitz invariant of $f^{\prime}$. This proves that the Hurwitz invariant is indeed an invariant of the modular group Hurwitz class of a NET map.

It remains to prove that if two modular group Hurwitz classes of NET maps have equal Hurwitz invariants, then they are equal. So let $f$ and $f^{\prime}$ be NET maps with postcritical sets $P_{2}$ and $P_{2}^{\prime}$ and equal Hurwitz invariants. So there exists an orientation-preserving affine 


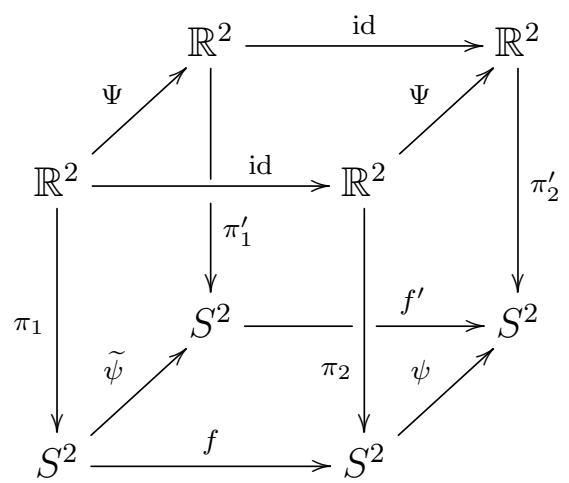

FiguRE 4. Proving the backward direction of Theorem 6.1

isomorphism $\Psi: \Lambda_{2} \rightarrow \Lambda_{2}^{\prime}$, which we extend to all of $\mathbb{R}^{2}$, such that $\Psi\left(\Lambda_{1}\right)=\Lambda_{1}^{\prime}$ and $\Psi$ induces an affine bijection $\bar{\Psi}: \mathcal{A} \rightarrow \mathcal{A}^{\prime}$ which takes the Hurwitz structure set $p_{1}^{-1}\left(P_{2}\right)$ of $f$ to the Hurwitz structure set $\left(p_{1}^{\prime}\right)^{-1}\left(P_{2}^{\prime}\right)$ of $f^{\prime}$. So Lemma 2.1 yields orientation-preserving homeomorphisms $\psi:\left(S^{2}, P_{2}\right) \rightarrow\left(S^{2}, P_{2}^{\prime}\right)$ and $\widetilde{\psi}:\left(S^{2}, P_{1}\right) \rightarrow\left(S^{2}, P_{1}^{\prime}\right)$ such that $\psi \circ \pi_{2}=\pi_{2}^{\prime} \circ \Psi$ and $\widetilde{\psi} \circ \pi_{1}=\pi_{1}^{\prime} \circ \Psi$. Hence every face of the cube of Figure 4 other than possibly the bottom is commutative. Since the vertical maps are surjective, the bottom is also commutative. Because $\Psi$ induces a map from the Hurwitz structure set of $f$ to the Hurwitz structure set of $f^{\prime}$, it follows that $\widetilde{\psi}\left(P_{2}\right)=P_{2}^{\prime}=\psi\left(P_{2}\right)$. Hence $f$ and $f^{\prime}$ are modular group Hurwitz equivalent.

This proves Theorem 6.1.

Corollary 6.2. The elementary divisors of a NET map $f$ are invariants of the modular group Hurwitz class of $f$.

Corollary 6.3. Every modular group Hurwitz class of NET maps is represented by a NET map whose presentation matrix is diagonal.

Corollary 6.4. In the typical cases, there is a bijection between the set of Hurwitz classes of NET maps with elementary divisors $(m, n)$ and the set of equivalence classes of Hurwitz structure sets in groups of the form $(\mathbb{Z} / 2 m \mathbb{Z}) \oplus(\mathbb{Z} / 2 n \mathbb{Z})$, were $m$ and $n$ are positive integers with $m \geq 2$ and $n \mid m$.

Remark 6.5. In the atypical cases, there are Hurwitz structure sets for which no Thurston map in the modular group Hurwitz equivalence class which they determine has four postcritical points.

Remark 6.6. Theorem 6.1 can be used to find one representative in every modular group Hurwitz class of NET maps of small degree. This is done in [15] through degree 30.

We next prove that the elementary divisors of a NET map form a complete set of invariants for topological equivalence. We note that Thurston equivalence, pure modular group Hurwitz equivalence, modular group Hurwitz equivalence and topological equivalence are successively weaker equivalence relations.

Theorem 6.7. Two NET maps are topologically equivalent if and only if their elementary divisors are equal. 
Proof. Let $f$ and $f^{\prime}$ be NET maps. We first prove the forward implication.

So suppose that $f$ and $f^{\prime}$ are topologically equivalent. Then there exist homeomorphisms $\varphi, \psi: S^{2} \rightarrow S^{2}$ such that $\varphi \circ f=f^{\prime} \circ \psi=\psi \circ\left(\psi^{-1} \circ f^{\prime} \circ \psi\right)$. Corollary 6.2 implies that $f^{\prime}$ and $\psi^{-1} \circ f^{\prime} \circ \psi$ have the same elementary divisors, so we may assume that $\varphi \circ f=\psi \circ f^{\prime}$. Combining $\varphi$ and $\psi$, we may even assume that $f^{\prime}=\varphi \circ f$. It follows that $\varphi$ maps the critical values of $f$ bijectively to the critical values of $f^{\prime}$. As discussed in the introduction, a NET map has exactly two critical values if and only if its elementary divisors are $(2,1)$ and it has exactly three critical values if and only if its elementary divisors are $(2,2)$. So we may assume that we are not in one of these atypical cases. Thus we have that $\varphi\left(P_{f}\right)=P_{f^{\prime}}$. Now let $\Lambda_{1}, \Lambda_{2}, p_{1}, q_{1}, p_{2}$ and $q_{2}$ be usual lattices and branched covering maps for $f$, so that $f \circ p_{1} \circ q_{1}=p_{2} \circ q_{2}$. Then

$$
f^{\prime} \circ p_{1} \circ q_{1}=\varphi \circ f \circ p_{1} \circ q_{1}=\left(\varphi \circ p_{2}\right) \circ q_{2} .
$$

This and the fact that $\varphi\left(P_{f}\right)=P_{f^{\prime}}$ imply that $\Lambda_{1}, \Lambda_{2}, p_{1}, q_{1}, \varphi \circ p_{2}$ and $q_{2}$ are corresponding lattices and branched covering maps for $f^{\prime}$. Since $\Lambda_{1}$ and $\Lambda_{2}$ determine the elementary divisors for both $f$ and $f^{\prime}$, the elementary divisors of $f$ and $f^{\prime}$ are equal. This proves the forward implication of Theorem 6.7.

To prove the backward implication, suppose that the elementary divisors of $f$ and $f^{\prime}$ are equal. Since every NET map is topologically equivalent to a Euclidean NET map and topologically equivalent NET maps have equal elementary divisors, we may assume that $f$ and $f^{\prime}$ are Euclidean. Since the translation term in a NET map presentation preserves equivalence, we may assume that $f$ and $f^{\prime}$ are induced by maps which are not just affine, but even linear. We may also assume that $f$ and $f^{\prime}$ have presentations with $\Lambda_{2}=\Lambda_{2}^{\prime}=\mathbb{Z}^{2}$. Now we apply the well-known result that if $A$ and $A^{\prime}$ are $2 \times 2$ matrices of integers with positive determinants and equal elementary divisors, then there exist $Q, R \in \mathrm{SL}(2, \mathbb{Z})$ such that $A^{\prime}=Q A R$. The matrices $Q$ and $R$ induce homeomorphisms of $S^{2}$ as in Lemma 2.1, which provide an equivalence between $f$ and $f^{\prime}$.

This proves Theorem 6.7 .

The definition of Hurwitz invariant given above has the virtue of expediency but not the virtue of naturality. The point here is that the definition of equivalence of Hurwitz structure sets in the finite group $\mathcal{A}$ relies on presenting the group as $\Lambda_{2} / 2 \Lambda_{1}$ and lifting to $\Lambda_{2}$ rather than working in $\mathcal{A}$ intrinsically. The rest of this section is devoted to giving another definition of equivalence of Hurwitz structure sets which does not rely on lifting to $\Lambda_{2}$.

For this we define the notion of special automorphism. Let $\mathcal{A}$ be a finite Abelian group generated by two elements. There exist unique positive integers $m$ and $n$ with $n \mid m$ such that $\mathcal{A} \cong(\mathbb{Z} / m \mathbb{Z}) \oplus(\mathbb{Z} / n \mathbb{Z})$. (The $m$ and $n$ here differ from the usual $m$ and $n$ by factors of 2.) Let $\varphi: \mathcal{A} \rightarrow \mathcal{A}$ be a group automorphism. Then $\varphi(n \mathcal{A})=n \mathcal{A}$, and so $\varphi$ induces an automorphism of $\mathcal{A} / n \mathcal{A} \cong(\mathbb{Z} / n \mathbb{Z}) \oplus(\mathbb{Z} / n \mathbb{Z})$. Automorphisms of the latter group are described by matrices just as over a vector space. In particular, determinants are meaningful. By choosing an isomorphism between $\mathcal{A} / n \mathcal{A}$ and $(\mathbb{Z} / n \mathbb{Z}) \oplus(\mathbb{Z} / n \mathbb{Z})$, the automorphism $\varphi$ induces an automorphism $\bar{\varphi}$ of $(\mathbb{Z} / n \mathbb{Z}) \oplus(\mathbb{Z} / n \mathbb{Z})$. The map $\bar{\varphi}$ depends on the choice of isomorphism between $\mathcal{A} / n \mathcal{A}$ and $(\mathbb{Z} / n \mathbb{Z}) \oplus(\mathbb{Z} / n \mathbb{Z})$, but its determinant does not. (This is analogous to changing bases of a vector space.) We say that $\varphi$ is a special automorphism if $\operatorname{det}(\bar{\varphi})=1$. We take this condition to be vacuously true when $n=1$.

Now that we have a definition, we can compute as follows. Choose an isomorphism between $\mathcal{A}$ and $(\mathbb{Z} / m \mathbb{Z}) \oplus(\mathbb{Z} / n \mathbb{Z})$. Let $u$ and $v$ be the elements of $\mathcal{A}$ such that $u$ corresponds to $(1,0)$ 
and $v$ corresponds to $(0,1)$. Let $\varphi: \mathcal{A} \rightarrow \mathcal{A}$ be a group automorphism. There exist integers $a, b, c, d$ so that $\varphi(x u+y v)=(a x+b y) u+(c x+d y) v$ for all integers $x$ and $y$. Then $\varphi$ is a special automorphism if and only if $a d-b c \equiv 1 \bmod n$.

Now suppose that our finite Abelian group has the form $\mathcal{A}=\Lambda_{2} / 2 \Lambda_{1}$, as usual, where $\Lambda_{2}=\mathbb{Z}^{2}$. Suppose that $\Phi: \Lambda_{2} \rightarrow \Lambda_{2}$ is an orientation-preserving affine isomorphism such that $\Phi\left(\Lambda_{1}\right)=\Lambda_{1}$. Then $\Phi(x)=A x+b$, where $A \in \mathrm{SL}(2, \mathbb{Z})$ and $b \in \Lambda_{1}$. So the map $x \mapsto A x$ induces a special automorphism of $\mathcal{A}$, and the map $x \mapsto x+b$ induces a translation by an element of order at most 2. Now we see how to define equivalence of Hurwitz structure sets in $\mathcal{A}$ without lifting to $\Lambda_{2}$ : Hurwitz structure sets $\mathcal{H S}$ and $\mathcal{H} \mathcal{S}^{\prime}$ in $\mathcal{A}$ are equivalent if and only if there exists a special automorphism $\varphi: \mathcal{A} \rightarrow \mathcal{A}$ and an element $b \in \mathcal{A}$ with $2 b=0$ such that $\mathcal{H S}^{\prime}=\varphi(\mathcal{H S})+b$. This definition coincides with the definition given above if every special automorphism of $\mathcal{A}$ lifts to $\Lambda_{2}$ because it is easy to lift the translation terms. This is the content of the next lemma. We have proved:

Theorem 6.8. The assignment $f \mapsto \mathcal{H S} \subset \mathcal{A}$ induces a bijection between modular Hurwitz classes of typical NET maps having elementary divisors $(m, n)$ and orbits of Hurwitz structure sets under the action of the group generated by the special automorphisms of $\mathcal{A} \cong(\mathbb{Z} / 2 m \mathbb{Z}) \oplus$ $(\mathbb{Z} / 2 n \mathbb{Z})$ and translations by elements of order 2.

Lemma 6.9. Let $m$ and $n$ be positive integers with $n \mid m$. Let $\varphi$ be a special automorphism of $(\mathbb{Z} / m \mathbb{Z}) \oplus(\mathbb{Z} / n \mathbb{Z})$. Then there exists an orientation-preserving group automorphism $\Phi: \mathbb{Z}^{2} \rightarrow$ $\mathbb{Z}^{2}$ which stabilizes the subgroup $\langle(m, 0),(0, n)\rangle$ and induces the map $\varphi$. To state this more concretely, let $a, b, c, d$ be integers such that $\varphi(1,0)=a(1,0)+c(0,1)$ and $\varphi(0,1)=$ $b(1,0)+d(0,1)$. Then there exists $\left[\begin{array}{ll}a^{\prime} & b^{\prime} \\ c^{\prime} & d^{\prime}\end{array}\right] \in S L(2, \mathbb{Z})$ such that $\left(a^{\prime}, b^{\prime}\right) \equiv(a, b) \bmod m$ and $\left(c^{\prime}, d^{\prime}\right) \equiv(c, d) \bmod n$.

Proof. We prove the concrete formulation of the lemma. In the situation of the concrete formulation, if $a=0$, then we may set $a=m$. Hence we may assume that $a \neq 0$. The assumptions imply that $a d-b c \equiv 1 \bmod n$.

Now we define an integer $b^{\prime}$ such that $b^{\prime} \equiv b \bmod m$, and in the next paragraph we prove that $\operatorname{gcd}\left(a, b^{\prime}\right)=1$. Because $a \neq 0$, only finitely many primes divide $a$. Using the Chinese remainder theorem, we find an integer $z$ such that for every prime $p$ dividing $a$ we have that

$$
z \equiv\left\{\begin{array}{lll}
0 & \bmod p & \text { if } p \nmid b \\
1 & \bmod p & \text { if } p \mid b
\end{array}\right.
$$

We set $b^{\prime}=b+z m$.

Now we show that $\operatorname{gcd}\left(a, b^{\prime}\right)=1$. If $p$ is a prime with $p \mid a$ and $p \nmid b$, then $p \mid z m$ and $p \nmid b^{\prime}$. It remains to consider primes $p$ such that $p \mid a$ and $p \mid b$. In this case $p \nmid n$ because $a d-b c \equiv 1 \bmod n$. On the other hand, if $p \mid \frac{m}{n}$, then $n \mid \frac{m}{p}$ and

$$
\frac{m}{p} \varphi(1,0)=\frac{m}{p} a(1,0)+\frac{m}{p} c(0,1)=0+0=0 .
$$

This is impossible because $\varphi(1,0)$ has order $m$. Hence $p \nmid n$ and $p \nmid \frac{m}{n}$, and so $p \nmid m$. Hence $p \nmid z m$, and so $p \nmid b^{\prime}$. Therefore $\operatorname{gcd}\left(a, b^{\prime}\right)=1$.

Since $\operatorname{gcd}\left(a, b^{\prime}\right)=1$, there exist integers $x$ and $y$ such that $a x+b^{\prime} y=1$. Set

$$
c^{\prime}=c-y\left(1-\left(a d-b^{\prime} c\right)\right), \quad d^{\prime}=d+x\left(1-\left(a d-b^{\prime} c\right)\right) \quad \text { and } \quad a^{\prime}=a .
$$


Then $\left(a^{\prime}, b^{\prime}\right) \equiv(a, b) \bmod m,\left(c^{\prime}, d^{\prime}\right) \equiv(c, d) \bmod n$ and

$$
\begin{aligned}
\left|\begin{array}{ll}
a^{\prime} & b^{\prime} \\
c^{\prime} & d^{\prime}
\end{array}\right| & =a\left(d+x\left(1-\left(a d-b^{\prime} c\right)\right)\right)-b^{\prime}\left(c-y\left(1-\left(a d-b^{\prime} c\right)\right)\right) \\
& =a d-b^{\prime} c+\left(a x+b^{\prime} y\right)\left(1-\left(a d-b^{\prime} c\right)\right)=1 .
\end{aligned}
$$

Thus we have a matrix in $\operatorname{SL}(2, \mathbb{Z})$ which satisfies the desired congruences. We take $\Phi$ to be the automorphism of $\mathbb{Z}^{2}$ whose matrix with respect to the standard basis is this matrix. It remains to show that $\Phi$ stabilizes $\Lambda=\langle(m, 0),(0, n)\rangle$. Because every element of $\Lambda$ is divisible by $n$, it is clear that the second coordinate of every element in $\Phi(\Lambda)$ is divisible by $n$. Because $\varphi(0,1)$ has order $n$, the integer $b^{\prime}$ must be divisible by $m / n$. Now we see that the first coordinate of every element in $\Phi(\Lambda)$ is divisible by $m$.

This proves Lemma 6.9.

\section{Computing the virtual multi-endomorphism}

In this section, we illustrate the calculation of the virtual multi-endomorphism in a concrete example.

We work with a NET map $f$ given by circling one of the large dots in Figure 5. Our computations are independent of which vertex is circled.

The diagram determines (i) an ordered basis of $\Lambda_{1}$ given by $\lambda_{1}=(6,0)$ and $\lambda_{2}=(0,1)$, and (ii) a fundamental domain for $\Gamma_{1}$, which is the parallelogram drawn. The vertices in this fundamental domain which map to elements of $P_{2}=P_{f}$ under the natural projection to $S_{1}^{2}$ are $(1,0),(11,0),(2,0),(10,0),(1,1),(11,1),(2,1)$, and $(10,1)$.

We identify the pure modular group with $G=\bar{\Gamma}(2)$. We next find additional congruence conditions defining the subgroup $G_{f}$ of pure modular group liftables as follows.

Suppose $M=\left[\begin{array}{ll}a & b \\ c & d\end{array}\right] \in \Gamma(2)$.

For $M$ to stabilize $\Lambda_{1}$, we must have that $M \lambda_{1} \in \Lambda_{1}$. This imposes no restrictions on $M$. It must also be true that $M \lambda_{2} \in \Lambda_{1}$. This inclusion is equivalent to the congruence $b \equiv 0 \bmod 6$. So if $M \in \Gamma(2)$, then $M$ stabilizes $\Lambda_{1}$ if and only if $b \equiv 0 \bmod 6$.

In addition to the condition that $M$ stabilizes $\Lambda_{1}$, we need that the map which $M$ induces on $\mathcal{A}=\Lambda_{2} / 2 \Lambda_{1}$ fixes the elements of our Hurwitz structure set $\mathcal{H} \mathcal{S}=\{ \pm(1,0), \pm(2,0), \pm(1,1), \pm(2,1)\}$ up to \pm 1 . An easy computation shows that $M$ takes $(1,0)$ and $(1,1)$ to themselves up to \pm 1 if and only if $a \equiv \pm 1 \bmod 12$ and $b \equiv 0 \bmod 12$. The corresponding conditions for $(2,0)$ and $(2,1)$ are then also satisfied. So $G_{f}$ contains the images in $\operatorname{PSL}(2, \mathbb{Z})$ of all such matrices $M$ in $\operatorname{SL}(2, \mathbb{Z})$ such that $a \equiv \pm 1 \bmod 12, b \equiv 0 \bmod 12, c \equiv 0 \bmod 2$ and $d \equiv a \bmod 12$. To compute $G_{f}$, in addition to considering multiplication by such matrices $M$, we must also consider multiplication by such matrices $M$ together with the translation by a vector $b$ in $2 \Lambda_{2} \cap\left\{0, \lambda_{1}, \lambda_{2}, \lambda_{1}+\lambda_{2}\right\}=\left\{0, \Lambda_{1}\right\}$. We find that this adds no more elements to $G_{f}$. We conclude that $G_{f}$ is the image in $\operatorname{PSL}(2, \mathbb{Z})$ of all matrices $M=\left[\begin{array}{ll}a & b \\ c & d\end{array}\right] \in \operatorname{SL}(2, \mathbb{Z})$ such that $a \equiv \pm 1 \bmod 12, b \equiv 0 \bmod 12, c \equiv 0 \bmod 2$ and $d \equiv a \bmod 12$.

The simplest nontrivial element $\varphi$ of $G_{f}$ is represented by the matrix $\left[\begin{array}{ll}1 & 0 \\ 2 & 1\end{array}\right]$. We now compute the image $\widetilde{\varphi}$ of $\varphi$ under the modular group virtual endomorphism.

A horizontal line in $\mathbb{R}_{1}^{2}$ which avoids $\mathbb{Z}^{2}$ maps to a simple closed curve in $S_{1}^{2}$ which separates two points of $P_{2}$ from the other two points of $P_{2}$. So the slope function $\mu_{f}$ of $f$ maps slope 0 to a slope. To evaluate $\mu_{f}(0)$, consider Figure 6. The line segment $a$ has ordinary slope 0 , Its endpoints map to $P_{2}$ and no element of its interior maps to $P_{1} \cup P_{2}$. Applying Theorem 5.3 of [4] with the line segment $S$ there equal to $a$, we find that $\mu_{f}(0)$ is the slope relative to 


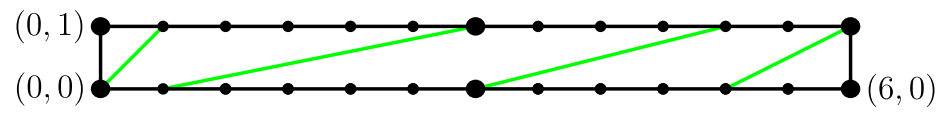

Figure 5. A virtual presentation diagram for $f$

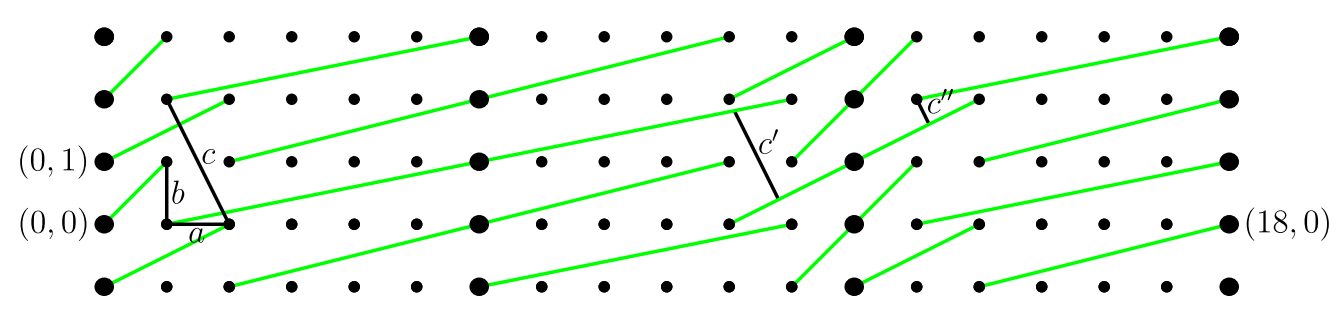

Figure 6. Computing $\mu_{f}$

the basis $\left(\lambda_{1}, \lambda_{2}\right)$ of the line segment joining the centers of the spin mirrors whose endpoints are the endpoints of $a$. The slope relative to $\left(\lambda_{1}, \lambda_{2}\right)$ of the line segment joining $(0,-1)$ and $(6,1)$ is 2 , so $\mu_{f}(0)=2$.

We compute $\mu_{f}(\infty)$ in the same way using the line segment $b$. The result is the slope relative to $\left(\lambda_{1}, \lambda_{2}\right)$ of the line segment joining $(0,0)$ and $(6,1)$, namely, 1 . We have the top of Figure 7 .

As in Section 6 of [6], we find that the pullback map $\mu_{\varphi}$ on slopes induced by $\varphi$ is given by the matrix $\left[\begin{array}{cc}1 & -2 \\ 0 & 1\end{array}\right]$. This yields the left side of Figure 7 .

In order to establish the bottom of Figure 7, we must evaluate $\mu_{f}(-2)$. For this we use line segment $c$ in Figure 6. This computation is a bit more complicated than for $a$ and $b$ because $c$ crosses two spin mirrors. We traverse $c$ starting at $(2,0)$. We meet the spin mirror centered at $(6,1)$ and spin to line segment $c^{\prime}$. We eventually meet the spin mirror centered at $(12,1)$ and spin to line segment $c^{\prime \prime}$. We conclude that $\mu_{f}(-2)$ is the slope relative to $\left(\lambda_{1}, \lambda_{2}\right)$ of the line segment joining $(0,-1)$ and $(18,3)$. Hence $\mu_{f}(-2)=\frac{4}{3}$. We now have the bottom of Figure 7 .

Because Figure 7 is commutative, the matrix representing $\mu_{\widetilde{\varphi}}$ is

$$
\left[\begin{array}{ll}
1 & 4 \\
1 & 3
\end{array}\right]\left[\begin{array}{ll}
1 & 2 \\
1 & 1
\end{array}\right]^{-1}=\left[\begin{array}{ll}
1 & 4 \\
1 & 3
\end{array}\right]\left[\begin{array}{cc}
-1 & 2 \\
1 & -1
\end{array}\right]=\left[\begin{array}{ll}
3 & -2 \\
2 & -1
\end{array}\right] .
$$

Because the upper right and lower left entries of this matrix are additive inverses of each other, this matrix also represents $\widetilde{\varphi}$.

According to NETmap, the degree of the map $X$ is 6 and the degree of the map $Y$ is 16 . Finding the algebraic curve defining the correspondence on moduli space discussed in the introduction will be difficult. 


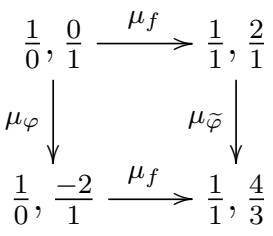

Figure 7. Computing $\mu_{\widetilde{\varphi}}$.

\section{BRANCH DATA}

Suppose $X, Y$ are closed oriented surfaces and $f: X \rightarrow Y$ is a finite orientation-preserving branched covering of degree $d \geq 2$. Let $C_{f}$ be the set of critical points of $f$, and let $V_{f}=f\left(C_{f}\right)$, the set of critical values of $f$. Generalizing the definition used in the introduction and $\S 6$, we say another such map $f^{\prime}: X^{\prime} \rightarrow Y^{\prime}$ is topologically equivalent to $f: X \rightarrow Y$ if there exist orientation-preserving homeomorphisms $\phi: X \rightarrow X^{\prime}, \psi: Y \rightarrow Y^{\prime}$ with $\psi \circ f=f^{\prime} \circ \phi$. Given $y \in Y$, the collection $\{\operatorname{deg}(f, x): f(x)=y\}$ of local degrees defines a partition of $d$. The branch data associated to $f$ is the set of such partitions for $y \in V_{f}$. Branch data is an invariant of topological equivalence.

Taking $X=Y=S^{2}$ and $f$ to be a NET map, the branch data of $f$ yields an invariant of the topological equivalence class of $f$. In this section, we study branch data associated to NET maps. Theorem 6.7 implies that the topological equivalence class of a NET map is determined by its elementary divisors. So the branch data of a NET map is computable from its elementary divisors. This will be made explicit after the proof of Lemma 8.1.

Since each critical point of a NET map has local degree 2, there is much redundancy in its branch data. It is therefore advantageous to focus on branch value preimages which are not critical points. From [4, Lemma 1.3], we know that the set $P_{1} \subseteq S^{2}-C_{f}$ of all noncritical points which $f$ maps to its postcritical set has exactly four points. Let $P_{1}^{*}=P_{1} \cap f^{-1}\left(V_{f}\right)$. The kernel of the branch data of $f$ is the set-theoretic data of the restriction $f \mid P_{1}^{*}: P_{1}^{*} \rightarrow$ $f\left(P_{1}^{*}\right)$, regarded as a function from one finite set to another.

Lemma 8.1. Suppose $f$ is a NET map. Let $(m, n)$ with $n \mid m$ be the elementary divisors of $f$. Exactly one of the following three cases occurs.

(1) $(m, n) \equiv(1,1)$ modulo $2 \Longleftrightarrow\left|V_{f}-f\left(P_{1}^{*}\right)\right|=0$

(2) $(m, n) \equiv(0,1)$ modulo $2 \Longleftrightarrow\left|V_{f}-f\left(P_{1}^{*}\right)\right|=2$

(3) $(m, n) \equiv(0,0)$ modulo $2 \Longleftrightarrow\left|V_{f}-f\left(P_{1}^{*}\right)\right|=3$

Proof. We first observe that $V_{f}-f\left(P_{1}^{*}\right)=P_{2}-f\left(P_{1}\right)$, where $P_{2}$ is the postcritical set of $f$. Up to topological equivalence, $f$ is the map on the sphere induced by the diagonal matrix $A$ with diagonal entries $(m, n)$. By Theorem 6.7, we may assume that $f$ has this form. The domain sphere is the double of an $m$-by- $n$ rectangle over its boundary; the codomain sphere is the double of the unit square over its boundary. The map $f$ folds in the obvious way. The set $P_{1}$ consists of the corners of the domain pillowcase. From this the lemma follows immediately.

A bit more formally: the set of corners $P_{1}$ of the domain sphere is naturally identified with the vector space $\Lambda_{1} / 2 \Lambda_{1}$; that of the codomain sphere with the vector space $\Lambda_{2} / 2 \Lambda_{2}$; the folding map induces a linear map between these vector spaces. Cases 1, 2, 3 then correspond respectively to the rank of this linear map being 2 , 1 , or 0 . 
The branch data of $f$ is easily reconstructed from knowledge of its degree $d$ and its branch data kernel: the kernel specifies precisely which partitions have a 1 as an additive factor, and how many times a 1 occurs. Here is the precise statement. In each partition, the number of 2 's is uniquely determined by the condition that the sum is the degree, $d$.

(1) $\left|V_{f}-f\left(P_{1}^{*}\right)\right|=0$; the partition over each element of $V_{f}$ is $\{2, \ldots, 2,1\}$

(2) $\left|V_{f}-f\left(P_{1}^{*}\right)\right|=2$; the partitions which contain 1's have the form $\{2, \ldots, 2,1,1\}$

(3) $\left|V_{f}-f\left(P_{1}^{*}\right)\right|=3$; the partition which contains 1's has the form $\{2, \ldots, 2,1,1,1,1\}$

In the typical case, exactly two partitions contain 1's in (2) and exactly one partition contains 1 's in (3). In the atypical case fewer partitions may contain 1's. Below, we refer to the three types of branch data by the same case numbers.

The question of which branch data arise from branched coverings-that is, are realizableis a classical, much-studied problem; see [17] and the references therein. The RiemannHurwitz formula gives an obvious necessary condition: here, this means that there are $2 d-$ 2 critical points, counted with multiplicity. We always assume that branch data satisfy this condition. The Riemann-Hurwitz conditions are not sufficient for branch data to be realizable. For example, the degree 6 branch data $\{2,1,1,1,1\}, 3 \times\{2,2,2\}$ is not realizable because statement 3 of Lemma 8.1 implies that 4 divides $d$ when $\left|V_{f}-f\left(P_{1}^{*}\right)\right|=3$. In general, [17, Thm. 3.8] implies the following.

Theorem 8.2. Branch data of types 1 and 2 are always realizable. Branch data of type 3 is realizable if and only if $d \equiv 0$ modulo 4 . In each realizable case, the realizing map can be taken to be a Euclidean NET map.

Note that the extra condition in the hypothesis in Case 3 is indeed satisfied by branch data of such NET maps (Lemma 8.1).

\section{DynAmic PORTRAits}

This section deals with dynamic portraits of NET maps. Corollary 9.3 completely characterizes dynamic portraits of NET maps. Theorem 9.2 gives, in addition, information about their relationship with elementary divisors. Algorithms for computing the correspondence between dynamic portraits of NET maps and NET map presentations are presented in the next two sections. Table 2 gives the number of NET map dynamic portraits in every degree.

We begin by discussing dynamic portraits of general Thurston maps from here to Lemma 9.1. By a dynamic portrait of a Thurston map $f$ we mean a weighted directed graph $\Gamma$ whose vertices are in bijective correspondence with the points of $S^{2}$ which are either critical or postcritical points of $f$. There is a directed edge from a vertex of $\Gamma$ corresponding to $x \in S^{2}$ to a vertex of $\Gamma$ corresponding to $y \in S^{2}$ if and only if $y=f(x)$. This edge is assigned a weight, the positive integer $\operatorname{deg}(f, x)$.

Let $\Gamma$ be a finite directed graph whose edges are weighted with positive integers for which exactly one edge emanates from every vertex. We define critical points (vertices), critical values (vertices) and postcritical vertices of $\Gamma$ in the straightforward way. We define the incoming degree of a vertex $v$ of $\Gamma$ to be the sum of the weights of the edges with terminal vertex $v$. The Riemann-Hurwitz formula imposes conditions on the dynamic portrait of a Thurston map $f$ :

$$
\sum_{x \in C_{f}}(\operatorname{deg}(f, x)-1)=2 d-2
$$


where $C_{f}$ is the set of critical points of $f$ and $d=\operatorname{deg}(f)$. In the same way, we say that $\Gamma$ satisfies the Riemann-Hurwitz formula if there exists an integer $d \geq 2$ for which the analogous equation holds and the incoming degree of every vertex of $\Gamma$ is at most $d$. We say that a finite weighted directed graph $\Gamma$ satisfies the obvious conditions for the dynamic portrait of a Thurston map if exactly one edge emanates from every vertex, each of its vertices is either critical or postcritical and it satisfies the Riemann-Hurwitz condition for some integer $d \geq 2$, the degree of $\Gamma$.

Let $\Gamma$ be a finite weighted directed graph which satisfies the obvious conditions for the dynamic portrait of a Thurston map. We can assign branch data to $\Gamma$ just as we assign branch data to a Thurston map. We are interested in whether or not $\Gamma$ is realizable, that is, isomorphic to the dynamic portrait of a Thurston map. It is clear that if $\Gamma$ is realizable, then its branch data is realizable. The next lemma reduces the realizability of $\Gamma$ to the realizability of its branch data.

Lemma 9.1. Let $\Gamma$ be a finite weighted directed graph which satisfies the obvious conditions for the dynamic portrait of a Thurston map. Suppose that the branch data of $\Gamma$ is realizable by a Thurston map $f$. Then $\Gamma$ is realizable by a Thurston maps topologically equivalent to $f$.

Proof. Let $A$ be the set of vertices of $\Gamma$ and let $B$ be the set of vertices of $\Gamma$ with at least one incoming edge. We regard $A, B$ as disjoint sets. The edges of $\Gamma$ determine a surjective map $\phi: A \rightarrow B$. The identity map defines an injection $\iota: B \hookrightarrow A$. Denote by $C \subset A$ the set of critical points and $V:=\phi(C) \subset B$ the set of critical values. Let $S_{A}^{2}, S_{B}^{2}$ be distinct copies of the sphere. Suppose the branch data extracted from $\Gamma$ is realizable. By hypothesis there exist a Thurston map $f: S_{A}^{2} \rightarrow S_{B}^{2}$ and embeddings $C \hookrightarrow S_{A}^{2}$ and $V \hookrightarrow S_{B}^{2}$ such that, upon identifying elements of $C$ and of $V$ with their images in the respective spheres, we have $\left.\phi\right|_{C}=\left.f\right|_{C}$, and the local degrees at points in $C$ coming from the portrait and from $f$ coincide. We extend the embedding $V \hookrightarrow S_{B}^{2}$ arbitrarily to an embedding $B \hookrightarrow S_{B}^{2}$.

In this paragraph, we show that there is an extension of the embedding $C \hookrightarrow S_{A}^{2}$ to an embedding $A \hookrightarrow S_{A}^{2}$ such that $\left.\phi\right|_{A}=\left.f\right|_{A}$. Pick $b \in B$. If $\phi^{-1}(b) \cap(A-C)=\emptyset$, then there is nothing to do. Otherwise, let $\left\{a_{1}, \ldots, a_{k}\right\}=\phi^{-1}(b) \cap(A-C)$. The local degree at each $a_{i}$ is equal to 1 . By the local degree constraint on $\Gamma$ and the compatibility of both local and global degrees, $f^{-1}(b) \cap\left(S_{A}^{2}-C\right)$ contains at least $k$ elements. We choose an embedding $\left\{a_{1}, \ldots, a_{k}\right\} \hookrightarrow f^{-1}(b) \cap\left(S_{A}^{2}-C\right)$ (it is not necessarily surjective: the sum of the local degrees in every fiber of $\phi$ is at most but not necessarily equal to $\operatorname{deg}(f))$. Repeating this construction for each $b$ establishes the result.

Finally, we "close" the map $f: A \rightarrow B$ to obtain a realization of the dynamic portrait. Recall that we have an injection $\iota: B \rightarrow A$ induced by the identity map on the vertices in the portrait. We arbitrarily choose an orientation-preserving homeomorphism $h: S_{B}^{2} \rightarrow S_{A}^{2}$ which agrees with $\iota$. The composition $h \circ f$ is a Thurston map topologically equivalent to $f$ which realizes $\Gamma$.

Now we specialize to NET maps. A finite weighted directed graph $\Gamma$ satisfies the obvious conditions for the dynamic portrait of a NET map if it satisfies the obvious conditions with some additional restrictions: every edge weight is either 1 or 2 , and the postcritical set $P$ of $\Gamma$ has exactly four points.

Let $\Gamma$ be a finite weighted directed graph which satisfies the obvious conditions for the dynamic portrait of a NET map. In this paragraph we define the mod 2 elementary divisors $m, n \in\{0,1\}$ of $\Gamma$. Motivated by Lemma 8.1, let $V$ be the set of critical values of $\Gamma$. Let $k$ 


\begin{tabular}{r|ccccccc}
$d$ & 2 & 3 & 4 & 5 & 6 & 7 & 8 \\
\hline$n$ & 16 & 94 & 272 & 144 & 338 & 152 & 476 \\
\hline \hline$d \bmod 4, d \geq 9$ & 0 & 1 & 2 & 3 & & & \\
\hline$n$ & 483 & 153 & 353 & 153 & & &
\end{tabular}

TABLE 2. The number $n$ of dynamic portraits for NET maps of degree $d$

be the number of elements $x$ of $V$ whose incoming degree is the degree of $\Gamma$ and no edge of $\Gamma$ ending at $x$ has weight 1 . As in Lemma 8.1, we have that $k$ is either 0,2 or 3 . The $\bmod$ 2 elementary divisors $(m, n)$ of $\Gamma$ are defined to be either $(1,1),(0,1)$ or $(0,0)$ accordingly.

We continue to let $\Gamma$ be a finite weighted directed graph which satisfies the obvious conditions for the dynamic portrait of a NET map with degree $d$. We say that $\Gamma$ satisfies the exceptional condition in the following situation. If the mod 2 elementary divisors of $\Gamma$ are both 0 , then 4 divides $d$.

We are finally ready for the main theorem on dynamic portraits of NET maps.

Theorem 9.2. Let $\Gamma$ be a finite weighted directed graph which satisfies the obvious conditions for the dynamic portrait of a NET map with degree $d$. Let $m$ and $n$ be positive integers such that $n \mid m$ and $m n=d$. Then there exists a NET map with a dynamic portrait isomorphic to $\Gamma$ and elementary divisors $m$ and $n$ if and only if $m$ and $n$ are congruent modulo 2 to the mod 2 elementary divisors of $\Gamma$.

Corollary 9.3. Let $\Gamma$ be a finite weighted directed graph which satisfies the obvious conditions for the dynamic portrait of a NET map. Then $\Gamma$ is isomorphic to the dynamic portrait of a NET map if and only if $\Gamma$ satisfies the exceptional condition.

Proof. The point here is that if $\Gamma$ satisfies the exceptional condition, then there exist positive integers $m$ and $n$ congruent modulo 2 to the $\bmod 2$ elementary divisors of $\Gamma$ such that $n \mid m$ and $m n=d$.

Remark 9.4. Pascali and Petronio prove Corollary 9.3 for Euclidean NET maps in Theorem 3.8 of [17]. Our Theorem 9.2 gives additional information about elementary divisors. It implies that in general such a graph is isomorphic to the dynamic portrait of many NET maps which are not only mutually Thurston inequivalent but even topologically inequivalent. Using the algorithm in Section 10, it is possible to find a NET map with a given dynamic portrait. This is done in [15] for every dynamic portrait through degree 40.

Remark 9.5. It is not difficult to see that there is a global bound on the number of NET map dynamic portraits for a given degree. Table 2 gives the number $n$ of NET map dynamic portraits in each degree $d$.

Proof of Theorem 9.2. To prove the forward implication, suppose that $f$ is a NET map that realizes $\Gamma$ and has elementary divisors $(m, n)$. Then $f$ realizes the branch data of $\Gamma$. By Lemma 8.1, the mod 2 elementary divisors of $\Gamma$ are congruent to $(m, n)$ modulo 2.

To prove the backward implication, suppose that the mod 2 elementary divisors of $\Gamma$ are congruent modulo 2 to $(m, n)$. Let $g$ be a Euclidean NET map induced by a diagonal matrix with diagonal entries $m$ and $n$. The elementary divisors of $g$ are $m$ and $n$. Lemma 8.1 and the discussion following it show that the branch data of $g$ is determined by the modulo 2 congruence classes of $m$ and $n$. The branch data of $\Gamma$ is determined by its mod 2 elementary 
divisors in the same way. Hence the branch data of $\Gamma$ equals the branch data of $g$. Now Lemma 9.1 and Theorem 6.7 complete the proof of Theorem 9.2 .

Remark 9.6. A dynamic portrait has hyperbolic type if each cycle contains a critical point. For topological polynomials, any Thurston map whose dynamic portrait has hyperbolic type is unobstructed, i.e. equivalent to a complex polynomial [8, Theorem 10.3.9]. Kelsey [9] establishes a partial converse: for almost all polynomial dynamic portraits of non-hyperbolic type, there is an obstructed topological polynomial with this portrait. Our data show this association between portraits and obstructedness breaks down for NET maps: the modular Hurwitz class 31HClass7 (and many others) consists entirely of unobstructed maps, and there are non-critical postcritical points. Composing with translations, we may find within this class examples of unobstructed NET maps with a non-critical fixed postcritical point.

\section{Explicit CONSTRUCtion OF THE DYNAMic PORTRAit OF A NET MAP}

In this section we present an algorithm which constructs the dynamic portrait of a NET map from a presentation for it. Starting with a presentation, we determine what to do, and then we give a step-by-step algorithm. We conclude with an example.

Let $\left(A, b, \alpha_{1}, \alpha_{2}, \alpha_{3}, \alpha_{4}\right)$ be presentation data for a NET map $f$. As usual, this allows $f$ to be expressed as a composition $f=h \circ g$. We seek combinatorial analogs $\phi, \gamma$ and $\eta$ for $f, g$ and $h$. We will use these combinatorial analogs to construct a dynamic portrait for $f$.

To begin, let $\lambda_{1}$ and $\lambda_{2}$ be the columns of $A$. They generate a sublattice $\Lambda_{1}$ of $\Lambda_{2}=\mathbb{Z}^{2}$ with index $\operatorname{det}(A)=\operatorname{deg}(f)$. Each green line segment $\alpha_{i}$ contains exactly one element of $\Lambda_{1}$. This element of $\Lambda_{1}$ is an endpoint of $\alpha_{i}$. We view $\alpha_{i}$ as directed, with this endpoint its initial endpoint. The four initial endpoints of $\alpha_{1}, \alpha_{2}, \alpha_{3}, \alpha_{4}$ map bijectively to $\Lambda_{1} / 2 \Lambda_{1}$. Let $P_{1}=\Lambda_{1} / 2 \Lambda_{1}$. The set $P_{1}$ will contain the noncritical points of $\Gamma$. Let $P_{2}$ be the set of images in $\left(\Lambda_{2} / 2 \Lambda_{1}\right) /\{ \pm 1\}$ of the terminal endpoints of $\alpha_{1}, \alpha_{2}, \alpha_{3}, \alpha_{4}$. The set $P_{2}$ will be the postcritical set of $\Gamma$.

Now we define $\eta: P_{1} \rightarrow P_{2}$. Let $x \in P_{1}$. There exists a unique $i \in\{1,2,3,4\}$ such that $x$ is the image in $P_{1}$ of the initial endpoint of $\alpha_{i}$. Let $\eta(x)$ be the image in $P_{2}$ of the terminal endpoint of $\alpha_{i}$.

We define $\gamma: P_{1} \cup P_{2} \rightarrow P_{1}$ in this paragraph. There exist integers $b_{1}$ and $b_{2}$ such that $b=b_{1} \lambda_{1}+b_{2} \lambda_{2}$. For every $\lambda \in \Lambda_{1}$, let $\bar{\lambda}$ be the image of $\lambda$ in $\Lambda_{1} / 2 \Lambda_{1}$. To define $\gamma$, let $x \in P_{1} \cup P_{2}$. Let $(r, s)$ be a representative of $x$ in $\mathbb{Z}^{2}$. Set

$$
\gamma(x)=\left(r+b_{1}\right) \bar{\lambda}_{1}+\left(s+b_{2}\right) \bar{\lambda}_{2} \in P_{1} .
$$

Now that we have $\gamma$ and $\eta$, we define $\phi: P_{1} \cup P_{2} \rightarrow P_{2}$ so that $\phi=\eta \circ \gamma$. We can easily construct a dynamic portrait for $f$ from $\phi$ and $d=\operatorname{det}(A)=\operatorname{deg}(f)$.

Here is a step-by-step algorithm based on the above discussion for constructing a dynamic portrait for a NET map with presentation data $\left(A, b, \alpha_{1}, \alpha_{2}, \alpha_{3}, \alpha_{4}\right)$.

Step 1 Let $\lambda_{1}$ and $\lambda_{2}$ be the columns of $A$, let $\Lambda_{2}=\mathbb{Z}^{2}$ and let $\Lambda_{1}$ be the sublattice of $\Lambda_{2}$ generated by $\lambda_{1}$ and $\lambda_{2}$. Direct $\alpha_{i}$ so that the initial endpoint of $\alpha_{i}$ is contained in $\Lambda_{1}$ for $i \in\{1,2,3,4\}$. Let $\bar{\lambda}$ denote the image in $\Lambda_{1} / 2 \Lambda_{1}$ of $\lambda$ in $\Lambda_{1}$.

Step 2 Let $P_{1}=\Lambda_{1} / 2 \Lambda_{1}$ be the set of images in $\left(\Lambda_{2} / 2 \Lambda_{1}\right) /\{ \pm 1\}$ of the initial endpoints of $\alpha_{1}, \alpha_{2}, \alpha_{3}, \alpha_{4}$, and let $P_{2}$ be the set of images in $\left(\Lambda_{2} / 2 \Lambda_{1}\right) /\{ \pm 1\}$ of the terminal endpoints of $\alpha_{1}, \alpha_{2}, \alpha_{3}, \alpha_{4}$. 


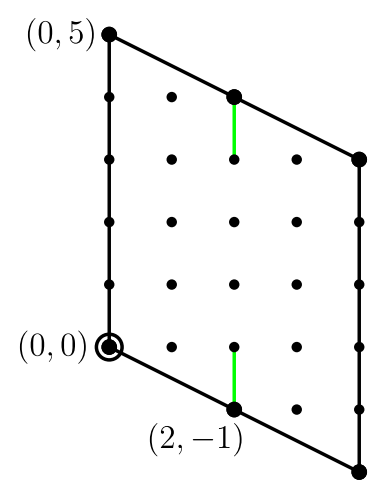

FiguRE 8. A presentation diagram for Example 10.1

Step 3 Define $\eta: P_{1} \rightarrow P_{2}$ so that $\eta$ maps the image in $P_{1}$ of the initial endpoint of $\alpha_{i}$ to the image in $P_{2}$ of the terminal endpoint of $\alpha_{i}$ for $i \in\{1,2,3,4\}$.

Step 4 Find the integers $b_{1}$ and $b_{2}$ such that $b=b_{1} \lambda_{1}+b_{2} \lambda_{2}$.

Step 5 For every $x \in P_{1} \cup P_{2}$ choose a representive $(r, s)$ for $x$ in $\mathbb{Z}^{2}$, and compute

$$
\gamma(x)=\left(r+b_{1}\right) \bar{\lambda}_{2}+\left(s+b_{2}\right) \bar{\lambda}_{2} \in \Lambda_{1} / 2 \Lambda_{1}=P_{1}
$$

Step 6 For every $x \in P_{2}$ construct an edge with initial vertex $x$ and terminal vertex $\phi(x)=$ $\eta(\gamma(x))$. This edge is given weight 1 if $x \in P_{1}$ and weight 2 if $x \in P_{2} \backslash P_{1}$.

Step 7 Adjoin enough new edges with weight 2, distinct initial endpoints not in $P_{1} \cup P_{2}$ and terminal endpoints in $P_{2}$ so that the incoming degree of every element $x$ of $P_{2}$ plus the order of $\phi^{-1}(x) \cap\left(P_{1}-P_{2}\right)$ is $d=\operatorname{det}(A)$.

Now we have our dynamic portrait.

Example 10.1. We illustrate this construction for the degree 10 NET map considered in Example 3.1 of [4]. Figure 8 has a presentation diagram for it. We write rows instead of columns to simplify notation.

We have that $A=\left[\begin{array}{cc}2 & 0 \\ -1 & 5\end{array}\right]$. The circled lattice point in Figure 8 is $(0,0)$, so $b=(0,0)$. The green line segments $\alpha_{1}$ and $\alpha_{3}$ are trivial, consisting of the points $(0,0)$ and $(0,5)$. The green line segment $\alpha_{2}$ joins $(2,-1)$ and $(2,0)$. The green line segment $\alpha_{4}$ joins $(2,4)$ and $(2,3)$.

Step $1 \lambda_{1}=(2,-1) \quad \lambda_{2}=(0,5)$

Step $2 P_{1}=\{\overline{(0,0)}, \overline{(2,-1)}, \overline{(0,5)}, \overline{(2,4)}\} \quad P_{2}=\{\overline{(0,0)}, \overline{(2,0)}, \overline{(0,5)}, \overline{(2,3)}\}$

Step $3 \eta(\overline{(0,0)})=\overline{(0,0)} \quad \eta(\overline{(2,-1)})=\overline{(2,0)} \quad \eta(\overline{(0,5)})=\overline{(0,5)} \quad \eta(\overline{(2,4)})=\overline{(2,3)}$

Step $4 b_{1}=0 \quad b_{2}=0$ 


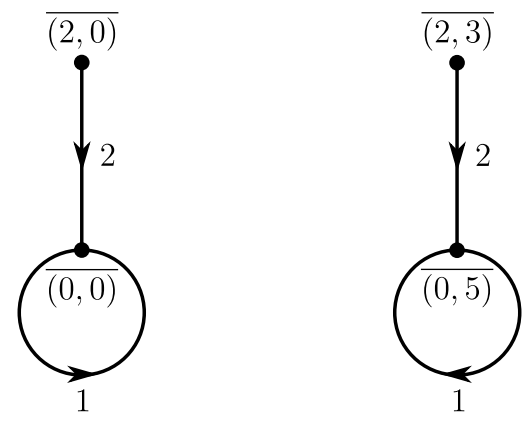

Figure 9. Step 6 for Example 10.1
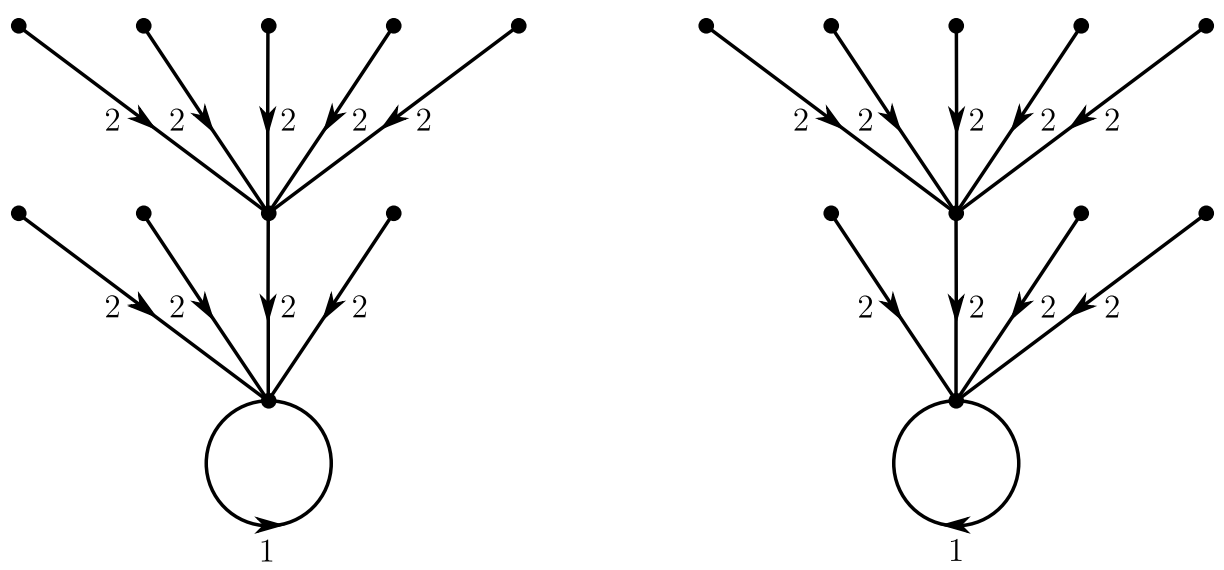

Figure 10. The dynamic portrait for Example 10.1

Step 5 We compute as follows.

$$
\begin{array}{cl}
\phi(\overline{(0,0)}) & =\eta(\gamma(\overline{(0,0)}))=\eta\left((0+0) \bar{\lambda}_{1}+(0+0) \bar{\lambda}_{2}\right)=\eta(0)=\overline{(0,0)} \\
\phi(\overline{(2,-1)}) & =\eta(\gamma(\overline{(2,-1)}))=\eta\left((2+0) \bar{\lambda}_{1}+(-1+0) \bar{\lambda}_{2}\right)=\eta\left(\bar{\lambda}_{2}\right)=\overline{(0,5)} \\
\phi(\overline{(0,5)}) & =\eta(\gamma(\overline{(0,5)}))=\eta\left((0+0) \bar{\lambda}_{1}+(5+0) \bar{\lambda}_{2}\right)=\eta\left(\bar{\lambda}_{2}\right)=\overline{(0,5)} \\
\phi(\overline{(2,4)}) & =\eta(\gamma(\overline{(2,4)}))=\eta\left((2+0) \bar{\lambda}_{1}+(4+0) \bar{\lambda}_{2}\right)=\eta(0)=\overline{(0,0)} \\
\phi(\overline{(2,0)}) & =\eta(\gamma(\overline{(2,0)}))=\eta\left((2+0) \bar{\lambda}_{1}+(0+0) \bar{\lambda}_{2}\right)=\eta(0)=\overline{(0,0)} \\
\phi(\overline{(2,3)}) & =\eta(\gamma(\overline{(2,3)}))=\eta\left((2+0) \bar{\lambda}_{1}+(3+0) \bar{\lambda}_{2}\right)=\eta\left(\bar{\lambda}_{2}\right)=\overline{(0,5)}
\end{array}
$$

Step 6 We construct a graph as in Figure 9 .

Step 7 We adjoin 16 critical vertices and corresponding edges to obtain Figure 10. This is essentially Figure 6 in [4].

\section{Explicit CONSTRUCTION OF NET MAPS FROM A DYNAMIC PORTRAit}

In this section we begin with a weighted directed graph $\Gamma$ which satisfies the obvious conditions for the dynamic portrait of a NET map and also the exceptional condition. We present an algorithm which constructs at least one representative of every Thurston equivalence class of NET maps with dynamic portrait isomorphic to $\Gamma$. This algorithm is essentially gotten 
by outlining the proofs of Lemma 8.1 and the backward implication of Theorem 9.2 . We conclude with an example.

Step 1 Determine the degree $d$ of $\Gamma$.

Step 2 Identify the set $P_{2}$ of postcritical vertices of $\Gamma$. Let $P_{1}$ be the set of noncritical vertices of $\Gamma$ together with more points disjoint from $\Gamma$ if necessary so that $\left|P_{1}\right|=4$. Define a map map $\phi: P_{1} \cup P_{2} \rightarrow P_{2}$ so that $\left.\phi\right|_{P_{2}}$ is the map determined by $\Gamma$ and $\left.\phi\right|_{P_{1}-P_{2}}$ is defined in any way so that the incoming degree of every element $x$ of $P_{2}$ plus the order of $\phi^{-1}(x) \cap\left(P_{1}-P_{2}\right)$ is $d$.

Step 3 Choose positive integers $m$ and $n$ such that $n \mid m, m n=d$ and $n$ is even if and only if the restriction of $\phi$ to $P_{1}$ is constant.

Step 4 Let $\Lambda_{2}=\mathbb{Z}^{2}$ and $\Lambda_{1}^{\prime}=\langle(m, 0),(0, n)\rangle$. Let $\Gamma_{1}^{\prime}$ be the group of Euclidean isometries of the form $x \mapsto 2 \lambda \pm x$ for some $\lambda \in \Lambda_{1}^{\prime}$. Let $F_{1}^{\prime}$ be the rectangle in $\mathbb{R}^{2}$ with corners $(0,0)$, $(2 m, 0),(0, n)$ and $(2 m, n)$. Identify $P_{1} \cup P_{2}$ with a subset of $\left(\Lambda_{2} / 2 \Lambda_{1}^{\prime}\right) /\{ \pm 1\}$ by labeling appropriate elements of $\Lambda_{2} \cap F_{1}^{\prime}$ with the elements of $P_{1} \cup P_{2}$. Then construct four directed line segments $\alpha_{1}^{\prime}, \alpha_{2}^{\prime}, \alpha_{3}^{\prime}, \alpha_{4}^{\prime}$ in $F_{1}^{\prime}$ whose images in $\mathbb{R}^{2} / \Gamma_{1}^{\prime}$ are disjoint such that

(1) $P_{1}=\{\overline{(0,0)}, \overline{(m, 0)}, \overline{(0, n)}, \overline{(m, n)}\}$;

(2) $\phi(x)=\phi(y)$ if and only if $x \equiv y \bmod 2 \Lambda_{2}$ for $x, y \in P_{1} \cup P_{2}$;

(3) the initial endpoint of $\alpha_{i}^{\prime}$ maps to $P_{1}$ and the terminal endpoint of $\alpha_{i}^{\prime}$ maps to $P_{2}$ for every $i \in\{1,2,3,4\}$.

Let $\eta: P_{1} \rightarrow P_{2}$ be the bijection which takes the image of the initial endpoint of $\alpha_{i}^{\prime}$ to the image of the terminal endpoint of $\alpha_{i}^{\prime}$ for every $i \in\{1,2,3,4\}$.

Step 5 The map $\eta^{-1} \circ \phi$ determines an injective function from a subset of $\Lambda_{2} / 2 \Lambda_{2}$ to $\Lambda_{1}^{\prime} / 2 \Lambda_{1}^{\prime}$. This injection extends to a bijection $\theta: \Lambda_{2} / 2 \Lambda_{2} \rightarrow \Lambda_{1}^{\prime} / 2 \Lambda_{1}^{\prime}$. There are 24 such bijections because $\left|\Lambda_{2} / 2 \Lambda_{2}\right|=\left|\Lambda_{1}^{\prime} / 2 \Lambda_{1}^{\prime}\right|=4$. Letting $\mathbb{F}_{2}$ denote the field of two elements, $\left|\mathrm{SL}\left(2, \mathbb{F}_{2}\right)\right|=6$ and $\left|\mathbb{F}_{2}^{2}\right|=4$. Hence there are $6 \times 4=24$ affine isomorphisms between two 2-dimensional vector spaces over $\mathbb{F}_{2}$. Thus $\theta$ is an affine isomorphism. Find $\bar{Q} \in \operatorname{SL}\left(2, \mathbb{F}_{2}\right)$ and $\bar{b}_{0} \in \mathbb{F}_{2}^{2}$ such that $\theta$ is given by $x \mapsto \bar{Q} x+\bar{b}_{0}$ with respect to the bases of $\Lambda_{2} / 2 \Lambda_{2}$ and $\Lambda_{1}^{\prime} / 2 \Lambda_{1}^{\prime}$ determined by the standard basis elements $e_{1}, e_{2}$ of $\Lambda_{2}$ and $m e_{1}, n e_{2}$.

Step 6 Choose $Q \in \mathrm{SL}(2, \mathbb{Z})$ and $b_{0} \in\left\{0, e_{1}, e_{2}, e_{1}+e_{2}\right\}$ such that $\bar{Q}$ and $\bar{b}_{0}$ are the reductions of $Q$ and $b_{0}$ modulo 2 .

Step 7 Set $A=Q\left[\begin{array}{cc}m & 0 \\ 0 & n\end{array}\right]$ and $b=A b_{0}$, where we view $b_{0}$ as a column vector.

Step 8 Define $F_{1}, \alpha_{1}, \alpha_{2}, \alpha_{3}, \alpha_{4}$ to be the images of $F_{1}^{\prime}, \alpha_{1}^{\prime}, \alpha_{2}^{\prime}, \alpha_{3}^{\prime}, \alpha_{4}^{\prime}$ under the map $x \mapsto Q x$.

The result is NET map presentation data $\left(A, b, \alpha_{1}, \alpha_{2}, \alpha_{3}, \alpha_{4}\right)$ for a NET map whose dynamic portrait is isomorphic to $\Gamma$.

Example 11.1. We illustrate this construction for the dynamic portrait in Figure 11.

Step $1 d=4$

Step $2 P_{1}=\left\{v_{2}, v_{8}, v_{9}, v_{10}\right\}, P_{2}=\left\{v_{2}, v_{3}, v_{4}, v_{5}\right\} \quad$ Three additional vertices $v_{8}, v_{9}, v_{10}$ are needed.

$$
\phi: \quad v_{1}, v_{3} \mapsto v_{2} \quad v_{2}, v_{4}, v_{8} \mapsto v_{3} \quad v_{5}, v_{9}, v_{10} \mapsto v_{4} \quad v_{6}, v_{7} \mapsto v_{5}
$$

Step $3 m=4, n=1$ 


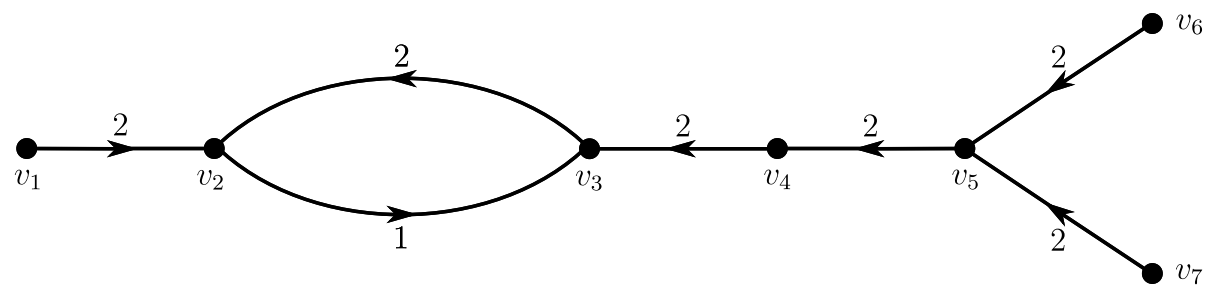

Figure 11. The dynamic portrait for Example 11.1

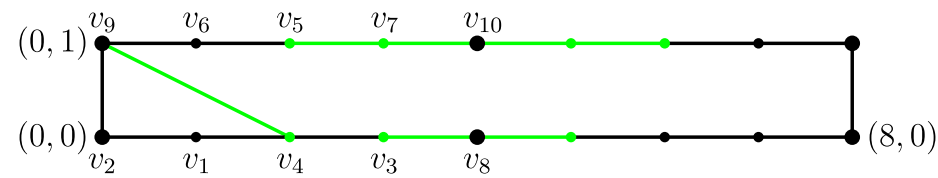

FigurE 12. The preliminary fundamental domain for Example 11.1

Step 4 We label appropriate elements of $\Lambda_{2} \cap F_{1}^{\prime}$ with the elements of $P_{1} \cup P_{2}$ as indicated in Figure 12. The critical points $v_{1}$ and $v_{3}$ are congruent modulo 2. The critical point $v_{4}$ and the noncritical points $v_{2}$ and $v_{8}$ are congruent modulo 2. The critical point $v_{5}$ and the noncritical points $v_{9}$ and $v_{10}$ are congruent modulo 2 . The critical points $v_{6}$ and $v_{7}$ are congruent modulo 2. The nontrivial green arcs are drawn in Figure 12 .

$$
\eta: \quad v_{2} \mapsto v_{2} \quad v_{8} \mapsto v_{3} \quad v_{9} \mapsto v_{4} \quad v_{10} \mapsto v_{5}
$$

Step 5 We evaluate $\eta^{-1} \circ \phi$ at three points.

$$
\begin{aligned}
& \overline{(0,0)}=v_{2} \stackrel{\phi}{\longmapsto} v_{3} \stackrel{\eta^{-1}}{\longmapsto} v_{8}=\overline{(4,0)}=1 \cdot \overline{m e_{1}}+0 \cdot \overline{n e_{2}} \\
& \overline{(1,0)}=v_{1} \stackrel{\phi}{\longmapsto} v_{2} \stackrel{\eta^{-1}}{\longmapsto} v_{2}=\overline{(0,0)}=0 \cdot \overline{m e_{1}}+0 \cdot \overline{n e_{2}} \\
& \overline{(0,1)}=v_{9} \stackrel{\phi}{\longmapsto} v_{4} \stackrel{\eta^{-1}}{\longmapsto} v_{9}=\overline{(0,1)}=0 \cdot \overline{m e_{1}}+1 \cdot \overline{n e_{2}}
\end{aligned}
$$

Let $\bar{Q}=\left[\begin{array}{ll}w & x \\ y & z\end{array}\right] \in \operatorname{SL}\left(2, \mathbb{F}_{2}\right)$.

$$
\begin{aligned}
\therefore & {\left[\begin{array}{ll}
w & x \\
y & z
\end{array}\right]\left[\begin{array}{l}
0 \\
0
\end{array}\right]+\bar{b}_{0}=\left[\begin{array}{l}
1 \\
0
\end{array}\right] \Longrightarrow \bar{b}_{0}=\left[\begin{array}{l}
1 \\
0
\end{array}\right] } \\
& {\left[\begin{array}{ll}
w & x \\
y & z
\end{array}\right]\left[\begin{array}{l}
1 \\
0
\end{array}\right]+\bar{b}_{0}=\left[\begin{array}{l}
0 \\
0
\end{array}\right] \Longrightarrow\left[\begin{array}{l}
w \\
y
\end{array}\right]+\left[\begin{array}{l}
1 \\
0
\end{array}\right]=\left[\begin{array}{l}
0 \\
0
\end{array}\right] \Longrightarrow w=1, y=0 } \\
& {\left[\begin{array}{ll}
w & x \\
y & z
\end{array}\right]\left[\begin{array}{l}
0 \\
1
\end{array}\right]+\bar{b}_{0}=\left[\begin{array}{l}
0 \\
1
\end{array}\right] \Longrightarrow\left[\begin{array}{l}
x \\
z
\end{array}\right]+\left[\begin{array}{l}
1 \\
0
\end{array}\right]=\left[\begin{array}{l}
0 \\
1
\end{array}\right] \Longrightarrow x=1, z=1 }
\end{aligned}
$$

Step 6 One choice for $Q$ and $b_{0}: Q=\left[\begin{array}{ll}1 & 1 \\ 0 & 1\end{array}\right], b_{0}=\left[\begin{array}{l}1 \\ 0\end{array}\right]$

Step $7 A=Q\left[\begin{array}{cc}m & 0 \\ 0 & n\end{array}\right]=\left[\begin{array}{ll}1 & 1 \\ 0 & 1\end{array}\right]\left[\begin{array}{ll}4 & 0 \\ 0 & 1\end{array}\right]=\left[\begin{array}{ll}4 & 1 \\ 0 & 1\end{array}\right], b=A b_{0}=\left[\begin{array}{l}4 \\ 0\end{array}\right]$

Step 8 Figure 13 has a presentation diagram for a NET map whose dynamic portrait is isomorphic to the initial portrait in Figure 11. 


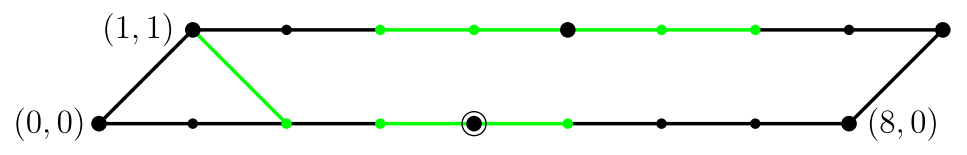

FiguRE 13. A presentation diagram for Example 11.1

\section{REFERENCES}

[1] L. Bartholdi and D. Dudko. Algorithmic aspects of branched coverings. https://arxiv.org/abs/1512. 05948, Submitted.

[2] L. Bartholdi and V. Nekrashevych. Thurston equivalence of topological polynomials. Acta. Math. 197(1), 2006, 1-51.

[3] X. Buff, A. Epstein, S. Koch, and K. Pilgrim, On Thurston's Pullback Map, in Complex Dynamics-Family and Friends, 561-583, Dierk Schleicher, ed. A K Peters/CRC Press, 2009.

[4] J. W. Cannon, W. J. Floyd, W. R. Parry and K. M. Pilgrim, Nearly Euclidean Thurston maps, Conform. Geom. Dyn. 16 (2012), 209-255 (electronic).

[5] Benson Farb and Dan Margalit, A Primer on Mapping Class Groups, Princeton Univ. Press, Princeton, 2012.

[6] W. Floyd, G. Kelsey, S. Koch, R. Lodge, W. Parry, K. M. Pilgrim, E. Saenz, Origami, affine maps, and complex dynamics. https://arxiv.org/abs/1612.06449. Submitted.

[7] W. J. Floyd, W. R. Parry and K. M. Pilgrim, Presentations of NET maps. https://arxiv.org/abs/ 1701.00443 . Submitted.

[8] J. H. Hubbard, Teichmüller theory, volume 2. Ithaca, NY: Matrix Editions, 2016.

[9] G. Kelsey, Mapping schemes realizable by obstructed topological polynomials Conform. Geom. Dyn. 16 (2012), 44-80.

[10] S. Koch, Teichmüller theory and critically finite endomorphisms, Advances in Mathematics Vol. 248, 2013.

[11] Sarah Koch, Kevin M. Pilgrim and Nikita Selinger, Pullback invariants of Thurston maps, Trans. Amer. Math. Soc. 368 (2016), no. 7, 4621-4655.

[12] R. Lodge. Boundary values of the Thurston pullback map. Conform. Geom. Dyn. 17 (2013), 77-118.

[13] T. Miyake, Modular Forms, Springer-Verlag, Berlin Heidelberg New York, 1989.

[14] V. Nekrashevych, Self-Similar Groups, Math. Surveys and Monographs 117, Amer. Math. Soc., Providence, 2005.

[15] The NET map website, www.math.vt.edu/netmaps/.

[16] W. Parry, NETmap, software, available from http://www.math.vt.edu/netmaps.

[17] M. A. Pascali and C. Petronio, Surface branched covers and geometric 2-orbifolds, Trans. Amer. Math. Soc. 361 (2009), 5885-5920.

Department of Mathematics, Virginia Tech, Blacksburg, VA 24061, USA

E-mail address: floyd@math.vt.edu

$U R L:$ http://www.math.vt.edu/people/floyd

E-mail address: walter.parry@emich.edu

Department of Mathematics, Indiana University, Bloomington, IN 47405, USA

E-mail address: pilgrim@indiana.edu 